\title{
MONOPOLES, VORTICES AND STRINGS: CONFINEMENT AND DECONFINEMENT IN 2+1 DIMENSIONS AT WEAK COUPLING
}

\author{
IAN I. KOGAN \\ Department of Physics, Theoretical Physics, 1 Keble Road, \\ Oxford $O X 13 N P, U K$ \\ ALEX KOVNER \\ Department of Mathematics and Statistics, University of Plymouth, 2 Kirkby Place, \\ Plymouth PL4 8AA, UK
}

We consider, from several complementary perspectives, the physics of confinement
and deconfinement in the $2+1$ dimensional Georgi-Glashow model. Polyakov's
monopole plasma and 't Hooft's vortex condensation are discussed first. We then
discuss the physics of confining strings at zero temperature. We review the Hamil-
tonian variational approach and show how the linear confining potential arises in
this framework. The second part of this review is devoted to study of the de-
confining phase transition. We show that the mechanism of the transition is the
restoration of 't Hooft's magnetic symmetry in the deconfined phase. The heavy
charged $W$ bosons play a crucial role in the dynamics of the transition, and we
discuss the interplay between the charged $W$ plasma and the binding of monopoles
at high temperature. Finally we discuss the phase transition from the point of
view of confining strings. We show that from this point of view the transition is
not driven by the Hagedorn mechanism (proliferation of arbitrarily long strings),
but rather by the "disintegration" of the string due to the proliferation of 0 branes.

\section{Contents}

1 Introduction 3

\begin{tabular}{|lll}
2 & The monopole plasma & 4
\end{tabular}

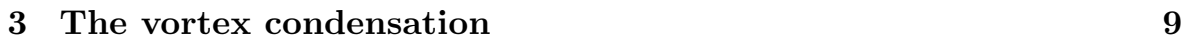

4 Confining strings 13

4.1 The Kalb-Ramond way . . . . . . . . . . . . . . . . . . . . . 14

4.2 The confining string for pedestrians . . . . . . . . . . . . . . 15

4.3 The string is soft ... and therefore rigid! . . . . . . . . . . . . . 16

4.4 The $W^{ \pm}$bosons - the 0 branes of the confining string . . . . . 20 
5 The Hamiltonian approach to compact U(1) 22

5.1 The variational ansatz $\ldots \ldots \ldots \ldots \ldots . \ldots \ldots$

5.2 Normalization integral . . . . . . . . . . . . . . . . . . . 25

5.3 Expectation values . . . . . . . . . . . . . . . . . . . 27

5.4 Determination of the ground state . . . . . . . . . . . 28

5.5 Spatial Wilson loops . . . . . . . . . . . . . . . . . . . 30

5.6 Interaction potential between external charges . . . . . . . . . 31

5.7 Relation between the Lagrangian and the Hamiltonian pictures 36

\begin{tabular}{|lll}
6 & The deconfining phase transition & 37
\end{tabular}

6.1 The monopole binding . . . . . . . . . . . . . 38

6.2 Magnetic symmetry restoration and the charged plasma . . . . 40

6.3 The renormalization group analysis . . . . . . . . . . . . . 43

6.4 The universality class of the phase transition $\ldots \ldots \ldots$. . . . 47

7 Vortices and monopoles at high temperature 48

8 Hot confining strings: Deconfinement versus Hagedorn transitions 52

8.1 The monopole binding as the Hagedorn transition . . . . . . . 53

8.2 Vortices on the world sheet - open strings and charged particles 55

9 The SU $(N)$ model $\quad 60$

9.1 The monopole-instantons and the Polyakov effective Lagrangian 63

9.2 The magnetic $Z_{N}$ symmetry $\ldots \ldots \ldots \ldots \ldots$

9.3 The deconfining transition $\ldots \ldots \ldots \ldots \ldots$

$\begin{array}{ll}10 \text { Concluding remarks } & 72\end{array}$ 


\section{Introduction}

Study of the confining regime in QCD is one of the most elusive and complicated subjects in modern particle physics. The theory is strongly interacting, and our arsenal as of today does not contain any reliable analytical tools to tackle strongly interacting quantum field theory. The situation is exacerbated in QCD by the fact that confinement is not the only nonperturbative phenomenon that plays out in the QCD vacuum. The spontaneous breaking of approximate chiral symmetry is the other important player. Although we believe that in principle the two phenomena are quite distinct, in the framework of QCD they are not easily separable. It is thus very important to have a simpler setting where one can understand the physics of confinement without having to worry about other complicated phenomena.

Thankfully, we do know a model, or rather a class of models, which exhibit confinement but are free from other complications of QCD. Those are weakly interacting confining gauge theories in $2+1$ dimensions. They were first studied by Polyakov almost twenty years ago. By now we understand the confining physics of these theories very well and have several perspectives on it. These results have been confirmed by numerical methods of lattice gauge theory Moreover in the last couple of years the finite temperature behavior of these theories has also been well understood, including the details of the deconfining phase transition.

Our aim in this review is to discuss in detail, and from several complementary points of view, the physics of these theories. We start in Sec. 2 with the description of the simplest of these models - the SU(2) gauge theory with the adjoint Higgs field - the Georgi-Glashow model. We describe Polyakov's derivation of confinement in this model based on the famous monopole plasma mechanism. In Sec. 3 we explain how confinement arises from the point of view of condensation of magnetic vortices. We show how the two pictures are closely related, but also point to some subtle differences between them and their complementary aspects. This has been discussed in detail 3 in a previous volume in this series, and so our discussion will be brief. Section 4 is devoted to the discussion of Polyakov's confining string. We explain how the rigidity of the mathematical string arises from the absence of "stringy" degrees of freedom in the ultraviolet. We also explain why the $W$ bosons play the role of D0 branes in the theory of the confining string. In Sec. 5 we show how the physics of confinement can be understood using Hamiltonian methods. We present a gauge invariant Hamiltonian variational calculation of the ground state wave function as well as of the potential between external sources. Section 6 is devoted to a detailed discussion of the deconfinement phase transition and the 
calculation of critical exponents. In Sec. 7 we discuss the properties of the vortex correlation functions and of the monopole interactions in the high temperature phase. We show that the monopoles are linearly "confined", while the vortex correlators decrease exponentially with distance. Section 8 discusses the same phase transition from the perspective of the confining string. It is shown that the would be "monopole binding" transition corresponds to the Hagedorn transition in the string language. The actual mechanism of the deconfining transition is the disintegration of the string due to proliferation of D0 branes. Section 9 presents the generalization of various aspects of the preceding discussion to the $\mathrm{SU}(N)$ Higgs model. Finally, Sec. 10 contains some concluding remarks.

\section{The monopole plasma}

We start our discussion of confinement in $2+1$ dimensions by recapitulating the original derivation of Polyakov. Consider the $2+1$ dimensional SU(2) gauge theory with a scalar field in the adjoint representation,

$$
S=-\frac{1}{2} \int d^{3} x \operatorname{tr}\left(F_{\mu \nu} F^{\mu \nu}\right)+\int d^{3} x\left[\frac{1}{2}\left(D_{\mu} h^{a}\right)^{2}+\frac{\lambda}{4}\left(h^{a} h^{a}-v^{2}\right)^{2}\right] .
$$

We adopt the notation

$$
A_{\mu}=\frac{i}{2} A_{\mu}^{a} \tau^{a}, \quad F_{\mu \nu}=\partial_{\mu} A_{\nu}-\partial_{\nu} A_{\mu}+g\left[A_{\mu}, A_{\nu}\right], \quad h=\frac{i}{2} h^{a} \tau^{a},
$$

and

$$
D_{\mu} h=\partial_{\mu} h+g\left[A_{\mu}, h\right] .
$$

The trace is normalized as

$$
\operatorname{tr}\left(\tau^{a} \tau^{b}\right)=2 \delta^{a b}
$$

We concentrate on the weakly coupled regime $v \gg g^{2}$. Since the coupling is weak one can try one's hand first at perturbation theory. Perturbatively the $\mathrm{SU}(2)$ gauge group is broken to $\mathrm{U}(1)$ by the large expectation value of the Higgs field. The photon associated with the unbroken subgroup is massless whereas the Higgs and the other two gauge bosons $W^{ \pm}$are heavy with the masses

$$
M_{H}^{2}=2 \lambda v^{2}, \quad M_{W}^{2}=g^{2} v^{2} .
$$

Thus, perturbatively the theory behaves very much like electrodynamics with spin one charged matter. However, this is not the whole story. It is known 
rigorously that the weakly coupled regime in this model is not separated by a phase transition from the confining regime, which one naturally identifies with the vanishing Higgs expectation value. In the confining regime the spectrum of the theory is certainly massive, and the charged particles are linearly confined. Thus, there must be some nonperturbative effects that bring about these two changes even at weak coupling. The nonperturbative effects in question are the monopoles, as first discussed by Polyakovi

It is easy to see that the Euclidean equations of motion of the model (11) have monopole like solutions,

$$
D_{\mu} F^{\mu \nu}=g\left[h, D^{\nu} h\right], \quad D_{\mu} D^{\mu} h=-\lambda\left(h^{a} h^{a}-v^{2}\right) h .
$$

The perturbative vacuum manifold is $\mathrm{SO}(3) / \mathrm{U}(1)$ and this has a non-zero second homotopy group, $\Pi_{2}(\mathrm{SO}(3) / \mathrm{U}(1))=Z$. Thus, the equations admit topologically nontrivial solutions with winding of the Higgs field at the spacetime infinity. The "elementary" configuration of this type is the well-known "t Hooft-Polyakov monopole,

$$
\begin{aligned}
h^{a}(\vec{x}) & =\hat{x}^{a} h(r), \\
g A_{\mu}^{a}(\vec{x}) & =\frac{1}{r} \epsilon_{a \mu \nu} \hat{x}^{\nu}(1-\phi(r),
\end{aligned}
$$

where $\hat{x}^{a}=x^{a} / r$. The non-Abelian field strength is

$$
g F_{\mu \nu}^{a}=\frac{1}{r^{2}} \epsilon_{\mu \nu b} \hat{x}^{a} \hat{x}^{b}\left(\phi^{2}-1\right)+\frac{1}{r}\left(\epsilon_{a \mu \nu}-\epsilon_{\mu \nu b} \hat{x}^{a} \hat{x}^{b}\right) \phi^{\prime},
$$

where the prime denotes differentiation with respect to the $3 \mathrm{D}$ radial coordinate $r$. The boundary conditions satisfied by the various fields are $h(0)=0$, $h(\infty)=v, \phi(0)=1$ and $\phi(\infty)=0$. The magnetic field far away from the origin is radial in the color space, and has a typical monopole-like behavior, dying away as $1 / r^{2}$. In fact, the Abelian field strength

$$
F_{\mu \nu}=\hat{h}^{a} F_{\mu \nu}^{a}-\frac{1}{g} \epsilon_{a b c} \hat{h}^{a}\left(D_{\mu} \hat{h}\right)^{b}\left(D_{\nu} \hat{h}\right)^{c}
$$

where

$$
\hat{h}^{a}=\frac{h^{a}}{|h|}
$$

is precisely that of a magnetic monopole everywhere except a small region around the origin - the monopole core. The core size is of the order of the vector meson mass. At weak coupling the action of the monopole solution is very large

$$
S_{\text {monopole }}=\frac{2 \pi M_{W}}{g^{2}} \epsilon\left(\frac{M_{H}}{M_{W}}\right)
$$


where

$$
\epsilon\left(\frac{M_{H}}{M_{W}}\right)
$$

is a slowly varying function such that $1 \leq \epsilon \leq 1.787$ (further details can be found in Ref. 5), and $\epsilon(\infty)=1$. Due to this large value of the action the contribution of the monopoles to the partition function is very small. Thus, typical configurations contain only very low density of monopoles and this dilute "monopole plasma" can be treated reliably in the fugacity expansion.

The monopoles interact via the three-dimensional Coulomb potential. The classical action for a configuration that contains far separated monopoles and anti-monopoles is

$$
S=\frac{2 \pi}{g^{2}} \sum_{a \neq b} \frac{q_{a} q_{b}}{\left|\vec{r}_{a}-\vec{r}_{b}\right|}+\frac{2 \pi M_{W}}{g^{2}} \epsilon\left(\frac{M_{H}}{M_{W}}\right) \sum_{a} q_{a}^{2}
$$

where $q_{a}= \pm 1$. The monopole fugacity is determined as

$$
\xi=\exp \left[-\frac{2 \pi M_{W}}{g^{2}} \epsilon\left(\frac{M_{H}}{M_{W}}\right)\right] .
$$

Even though this fugacity is tiny, the monopoles have a decisive effect on the low-energy properties of the theory. Since the $W$ bosons are very heavy, one can neglect their effects with good accuracy. In this approximation, the partition function of the theory factorizes into the partition function of the massless photon and that of the monopole plasma. The two factors can be combined into the partition function of a "dual photon" field. Following Polyakov's Derivationt we start from the statistical sum $Z_{M}$ for Coulomb plasma with $N_{+}$positive and $N_{-}$negative charges with equal fugacities $\xi$, which is given by

$$
Z_{\text {plasma }}=\sum_{N_{+}, N_{-}} \int \prod_{a=1}^{N_{+}} d^{3} \vec{r}_{a} \prod_{b=1}^{N_{-}} d^{3} \vec{r}_{b} \mu^{3\left(N_{+}+N_{-}\right)} \frac{1}{N_{+} ! N_{-} !} \exp (-S\{a, b\})
$$

where $\vec{r}_{a}$ and $\vec{r}_{b}$ are coordinates for positive and negative charges respectively. The dimensionful constant $\mu$ is necessary to convert the summation over the monopole coordinates into the integration. It is nonuniversal, but clearly is related to the shortest distance scale at which the present approximation is valid, namely the size of the monopole core. In Polyakov's calculation $\mu$ is determined to be

$$
\mu^{3} \propto \frac{M_{W}^{7 / 2}}{g} .
$$


Using the fact that the Coulomb potential is the inverse of the Laplacian operator, this can be written as 6

$$
\begin{aligned}
& Z_{\text {plasma }}=Z_{0}^{-1} \int D \phi \sum_{N_{+}, N_{-}} \int \prod_{a=1}^{N_{+}} d^{3} \vec{r}_{a} \prod_{b=1}^{N_{-}} d^{3} \vec{r}_{b} \frac{\left(\mu^{3} \xi\right)^{\left(N_{+}+N_{-}\right)}}{N_{+} ! N_{-} !} \\
& \exp \left(-\int d x \frac{g^{2}}{32 \pi^{2}}\left(\partial_{\mu} \phi\right)^{2}-\sum_{a, b} i \phi(x)\left[\left(\delta\left(x-x_{a}\right)-\delta\left(x-x_{b}\right)\right)\right]\right) .
\end{aligned}
$$

Here $Z_{0}$ is the partition function of a single massless field. In the dilute gas approximation, that is allowing at most one monopole or antimonopole at each spatial point, the summation over the coordinates is easily performed and one obtains for the partition function of the theory

$$
Z=\int D \phi \exp \left[-\int d^{3} x \mathcal{L}_{\text {eff }}\right]
$$

with the effective Lagrangian

$$
\mathcal{L}_{\text {eff }}=\frac{g^{2}}{32 \pi^{2}}\left(\partial_{\mu} \phi\right)^{2}-\xi \mu^{3} \cos \phi .
$$

Note that the factor $Z_{0}^{-1}$ in Eq. (13) cancelled against the contribution of a free massless photon of the Georgi-Glashow model.

One striking effect of the monopole plasma is explicit in this representation. The field $\phi$ which in Eq. (14) represents the photon, is not massless. Rather it has an exponentially small mass, determined by the monopole fugacity,

$$
M_{\gamma}^{2}=\frac{16 \pi^{2} \mu^{3} \xi}{g^{2}} .
$$

The other effect is confinement, or area law for the fundamental Wilson loop.

Let us calculate the expectation value of the Abelian Wilson loop

$$
W(C)=\left\langle\exp \left(i g \int_{\Sigma} d^{2} x B(x)\right)\right\rangle,
$$

where $B$ is a component of the Abelian magnetic field (6) and $\Sigma$ is a surface bounded by the curve $C$.

To calculate the expectation value, we insert this exponential factor into the path integral for the partition function of the model and use the fact that for a monopole plasma configuration

$$
\exp \left(i g \int_{\Sigma} d^{2} x B(x)\right)=\exp \left(i \int d^{3} x \eta(x) \rho(x)\right) .
$$


Here

$$
\eta(x)=\int_{\Sigma} \frac{(x-y)_{\mu}}{|x-y|^{3}} d^{2} \sigma_{\mu}(y)
$$

is the flux (normalized at $4 \pi$ ) through the surface $\Sigma$ from a monopole located at the point $x$, and

$$
\rho(x)=\sum_{a, b}\left[\delta\left(x-x_{a}\right)-\delta\left(x-x_{b}\right)\right]
$$

is the density of the magnetic charge in the plasma. As far as the summation over the monopole coordinates is concerned this is exactly the same type of term as the linear term in $\phi$ in the exponential in Eq. (13). Thus, the summation can again be performed, and one obtains

$$
W(C)=\frac{1}{Z} \int D \phi \exp \left[-\int d^{3} x \mathcal{L}_{\text {eff }}(\eta)\right]
$$

where

$$
\mathcal{L}_{\text {eff }}(\eta)=\frac{g^{2}}{32 \pi^{2}}\left(\partial_{\mu} \phi\right)^{2}-\frac{M_{\gamma}^{2} g^{2}}{16 \pi^{2}} \cos (\phi+\eta) .
$$

For large contours $C$ the path integral over $\phi$ can be calculated now in the steepest descent approximation. It is convenient to change variables to $\Phi=\phi+\eta$. The integral then is determined by the solution of classical equations of motion

$$
\partial^{2}(\Phi-\eta)=M_{\gamma}^{2} \sin (\Phi)
$$

The nature of this solution is easy to understand. Let us for definiteness imagine that the surface $\Sigma$ lies in the 1-2 plane. The Laplacian of $\eta$ vanishes everywhere in space, except on the surface $\Sigma$, where it is equal to the transverse derivative of the delta function with the coefficient $2 \pi$,

$$
\partial^{2} \eta(x)=2 \pi \partial_{z} \delta^{3}(x-\Sigma) .
$$

Thus, the field $\Phi$ satisfies the free sine-Gordon equation everywhere in space, except on the surface $\Sigma$. The presence of $\eta$ imposes the boundary condition that this particular solution must be discontinuous across $\Sigma$ with a discontinuity of $2 \pi$. Such a solution is easily constructed from a domain wall solution of the sine-Gordon theory. At negative $z$ the solution $\Phi$ is just the domain wall, so that at $z=0+$ it reaches the value $\pi$. It then jumps discontinuously to $-\pi$ on the other side of the surface, $\Phi(z=0-)=-\pi$ and rises back to zero at large positive $z$ again following the domain wall solution. The action of such a solution is obviously just equal to the action of a domain wall in the 
sine-Gordon model. It is proportional to the total area of the surface $\Sigma$, and the coefficient parametrically is given by $g^{2} M_{\gamma}$. Thus, we find

$$
W(C) \sim \exp (-\sigma S)
$$

where $S$ is the minimal area and $\sigma \sim g^{2} M_{\gamma}$. The Wilson loop therefore has an area law and the theory is confining.

Thus, the monopole plasma accounts nicely for the finite mass of the photon as well as for the area law of the Wilson loop.

\section{The vortex condensation}

A complementary view of confinement in this theory is the spontaneous break ing of the magnetic $Z_{2}$ symmetry due to the condensation of magnetic vortices 89 This approach has been reviewed in Ref. 3 and therefore we will be brief here.

The Georgi-Glashow model has a global $Z_{2}$ symmetry. This symmetry is not recognizable as a Noether type symmetry in the Lagrangian (11), but rather is akin to "topological" symmetry. It is generated by the total magnetic flux. Ignoring for the moment monopole effects, the Abelian magnetic field (6) is a conserved current

$$
\tilde{F}^{\mu}=\frac{1}{4} \epsilon_{\mu \nu \lambda} F_{\nu \lambda} .
$$

However, due to the monopole contributions (see Ref. 10), this symmetry is anomalous, and only the $Z_{2}$ subgroup is conserved. The non-anomalous $Z_{2}$ magnetic symmetry group is generated 11 by the large spatial Wilson loop which encloses the system,

$$
W(C \rightarrow \infty)=\exp \left(\frac{i g}{2} \int d^{2} x B(x)\right) .
$$

The order parameter for this $Z_{2}$ transformation is the operator that creates 10 an elementary magnetic vortex of flux $2 \pi / g$,

$$
V(x)=\exp \frac{2 \pi i}{g} \int_{C} \epsilon_{i j} \hat{h}^{a} E_{j}^{a}(x) .
$$

This operator is local, gauge invariant and a Lorentz scalar. Together with the spatial Wilson loop it forms the order-disorder algebra,

$$
W(C \rightarrow \infty) V(x)=-V(x) W(C \rightarrow \infty) .
$$

The vortex operator condenses in the vacuum and its expectation value is determined 3 by the gauge coupling constant $\langle V\rangle^{2}=g^{2} / 8 \pi^{2}$. 
Since the anomalous effects that break the U(1) magnetic symmetry down to $Z_{2}$ at weak coupling are small, one can construct the low-energy effective theory Lagrangian in terms of the relevant order parameter. It is given by the $Z_{2}$ invariant theory of the vortex field $V$,

$$
\mathcal{L}_{\text {eff }}=\partial_{\mu} V \partial^{\mu} V^{*}-\lambda\left(V V^{*}-\frac{g^{2}}{8 \pi^{2}}\right)^{2}+\frac{M^{2}}{4}\left\{V^{2}+\left(V^{*}\right)^{2}\right\}
$$

The vortex self-coupling in this effective Lagrangian can be determined as 12

$$
\lambda=\frac{2 \pi^{2} M_{W}^{2}}{g^{2}} .
$$

Note that as a low-energy Lagrangian, Eq. 28) is indeed consistent with Eq. (14). At weak gauge coupling, the quartic coupling $\lambda$ is very large. In this nonlinear $\sigma$-model limit the radius of the field $V$ is therefore frozen to its expectation value. The only relevant degree of freedom is the phase

$$
V(x)=\frac{g}{\sqrt{8} \pi} e^{i \chi} .
$$

Substituting this into Eq. (28) one indeed obtains precisely Eq. (14) with the identification $\phi=2 \chi$. Thus, Polyakov's dual photon is nothing but the pseudoGoldstone boson of the Lagrangian (28).

This relation can be inferred directly from the definition of $V$ in Eq. 26) and the monopole plasma partition function (13). Equation (13) relates the field $\phi$ with the monopole density as

$$
\frac{g^{2}}{16 \pi^{2}} \phi(x)=i \rho=\frac{g}{4 \pi} \partial_{\mu} \tilde{F}^{\mu}
$$

The factor $i$ has disappeared in the second equality since the field strength is now in Minkowski rather than Euclidean space. The factor $\frac{g}{4 \pi}$ is due to the fact that the minimal magnetic monopole charge is twice the fundamental value allowed by the Dirac quantization condition. This immediately gives

$$
\phi(x)=\frac{4 \pi}{g} \frac{\partial_{\mu}}{\partial^{2}} \tilde{F}^{\mu}=\frac{4 \pi}{g} \int_{x_{0}}^{x} d z_{\mu} \tilde{F}^{\mu}(z) .
$$

The integral on the right-hand side does not depend (to leading order in fugacity) on the choice of the integration curve. Choosing it to be at equal time and comparing to Eq. 26), we get

$$
\phi=2 \chi
$$


Thus, the non-vanishing photon mass is a natural consequence of the fact that the magnetic symmetry is discrete and not continuous, and thus the photon is a pseudo-Goldstone boson.

The calculation of the spatial Wilson loop in this framework closely follows the calculation in the previous subsection. For details we refer the reader to Ref. 3 .

Thus, as far as the description of the low-energy phenomena is concerned, the vortex approach is equivalent to the monopole plasma approach. The one conspicuous difference between them is that in the vortex low-energy approach the field $\chi$ is treated as a phase, while in the monopole plasma context $\phi$ is an ordinary real field which takes values on a straight line. As a consequence the Lagrangian (28) allows configurations with quantized discontinuities of the field $\chi$. In particular, the vortex configurations where the field $\chi$ winds around some point are allowed in the description of Eq. (28). In such a vortex configuration the field $\chi$ is discontinuous along an infinite cut. In the framework of the effective theory, Eq. (28), such a quantized discontinuity does not contribute to the energy. Polyakov's effective Lagrangian (14), on the other hand, does not allow such vortex configurations. Since the field $\phi$ in it is not treated as a phase, the cut does contribute to the kinetic energy term in Eq. (14), and this contribution is both ultraviolet and infrared divergent. The ultraviolet divergence is of no importance by itself, since the theory is defined with the intrinsic ultraviolet cutoff $M_{W}$. However, the infrared divergence indisputably puts the description of such vortex-like configurations beyond Eq. (14).

As it turns out this difference can sometimes be quite significant. The point is that these vortex-like configurations of the field $V$ are the low-energy incarnation of the charged states of the theory, the $W$ bosons.

As explained in Ref. 3, the electric current is identified with the topological current in Eq. (28),

$$
\frac{g}{\pi} J_{\mu}=i \epsilon_{\mu \nu \lambda} \partial_{\nu}\left(V^{*} \partial_{\lambda} V\right)
$$

Thus, charged states carry unit winding number of $\chi$. The $W^{+}\left(W^{-}\right)$boson is a state with positive (negative) unit winding of $\chi$.

Thus, the physics of the charged states cannot be discussed within Eq. (14), but can be considered within Eq. (28). Of course since the charged states are confined in this theory, the energy of an isolated charged state is linearly infrared divergent. The mechanism of this divergence, as well as the formation of the electric flux tube, is discussed in detail in Ref. 3. As we will see in later sections, proper treatment of the charged states is crucial for the understanding of the dynamics of the deconfinement phase transition and thus it is worthwhile to be careful on this point. We note that this is not the only instance in which 
the charged states play an important role. For example, as discussed in Ref. 13, the presence of these states leads to the breaking of the string of charge two, which in Polyakov's effective Lagrangian (14) is strictly stable.

In fact, to properly account for the large mass of the charged particles, the Lagrangian (28) must be slightly modified, since as it stands it underestimates their energy. The core energy of the soliton of $V$ should be equal to the mass of the charged vector boson $M_{W}$, since this is the lightest charged excitation in the theory. On the other hand, the core mass of a soliton in the Lagrangian (28) is given by the ultraviolet contribution of the Coulomb potential. With the cutoff of order $M_{W}$, this is of order $g^{2} \ln M_{W} / g^{2}$. This is not surprising, since the mass of the $W$ boson indeed comes from the distances of the order of its Compton wavelength, and is thus well inside the ultraviolet cutoff of the effective theory. The situation can be improved by adding to the effective Lagrangian a higher derivative term of the Skyrme type

$$
\delta L=\Lambda\left(\epsilon_{\mu \nu \lambda} \partial_{\nu} V^{*} \partial_{\lambda} V\right)^{2},
$$

with

$$
\Lambda \propto \frac{1}{g^{4}} \frac{1}{M_{W}} .
$$

The extra term Eq. (35) does not affect the photon mass nor the value of the string tension. The density of this action does not vanish only at the points where the phase of $V$ is singular - that is the points of winding. For a closed curve $C$ of length $L$ which carries the winding, the contribution of this extra term to the action is $M_{W} L$, which is precisely the action of the world line of a massive particle of mass $M_{W}$.

Thus, Polyakov's monopole plasma picture and 't Hooft's magnetic vortex condensate picture fit very neatly together and provide a fairly exhaustive understanding of confinement in the Georgi-Glashow model. Of course, both monopoles and vortices are not physical excitations of the Georgi-Glashow theory. What one would like to see is the confining string, the tube of electric flux. It is believed that in $3+1$ dimensional QCD at large $N_{c}$ these strings interact weakly and perhaps are described by a free string theory. Although one does not expect the same to be true in $2+1$ dimensions at $N_{c}=2$, the handle we have on the present model gives us a unique possibility to try and derive the relevant string theory. In the next section, therefore, we explain how one can derive in the long wavelength approximation the theory of the confining string. This theory turns out to have some quite peculiar features, which we will discuss. 


\section{Confining strings}

In 1996 Polyakov proposed 14 the derivation of the so-called confining string action explicitly from compact QED, which is equivalent to the Georgi-Glashow model in the low-energy approximation of Eq. (14). This action was studied further in Ref. 15 and it was found to describe a rigid string 16.17

We will first discuss the original derivation due to Polyakov which uses the Kalb-Ramond field 18 We will also give a more intuitive and simple derivation which uses the direct correspondence between field configurations and the summation over closed string world sheets. An advantage of this derivation is that it makes explicit an important and quite unusual property of the confining string, namely that the fluctuations of this string with large momenta (larger than the inverse thickness of the string) do not cost energy. This is a dynamical extension of the so called zigzag symmetry introduced by Polyakov 14 We also show that the heavy charged particles of the Georgi-Glashow model $\left(W^{ \pm}\right.$ bosons) appear as a certain kind of 0 branes in the string description.

Our starting point will be an expression for the Wilson loop discussed earlier,

$$
W(C)=\frac{1}{Z} \int D \phi \exp \left[-\int d^{3} x \mathcal{L}_{\mathrm{eff}}(\eta)\right]
$$

where

$$
\mathcal{L}_{\text {eff }}(\eta)=\frac{g^{2}}{32 \pi^{2}}\left(\partial_{\mu} \phi\right)^{2}-\frac{M^{2} g^{2}}{16 \pi^{2}} \cos (\phi+\eta) .
$$

Note that the effective theory at $\eta=0$ has more than one vacuum state. In particular, $\phi=2 \pi n$ for any integer $n$ is the classical ground state. Therefore, the classical equations of motion have wall-like solutions - where the two regions of space, say with $\phi=0$ and $\phi=2 \pi$ are separated by a domain wall. The action density per unit area of such a domain wall is parametrically

$$
\sigma \propto g^{2} M
$$

As we discussed in Sec. 2, the fundamental Wilson loop, when inserted into the path integral, induces such a domain wall solution, with the result

$$
\langle W(C)\rangle=\exp \{-\sigma S\}
$$

where $S$ is the minimal area subtended by the contour $C$. Thus, the string tension is precisely equal to the wall tension of the domain wall. This domain wall is identified with the world sheet of the confining QCD string. 


\subsection{The Kalb-Ramond way}

In order to find a string representation for $W(C)$ one can rewrite $W(C)$ in a different form, introducing a new auxiliary Kalb-Ramond field $B_{\mu \nu}$,

$$
W(C)=\int D B_{\mu \nu} D \phi \exp [-S(\phi, B)]
$$

with the action

$$
\begin{aligned}
S(\phi, B) & =\int d^{3} x\left[\frac{16 \pi^{2}}{g^{2}} B_{\mu \nu}^{2}+i \epsilon_{\mu \nu \rho} \partial_{\mu} \phi B_{\nu \rho}+\frac{M^{2} g^{2}}{16 \pi^{2}}(1-\cos \phi)\right] \\
& +i \int_{\Sigma_{C}} B_{\mu \nu} d \sigma_{\mu \nu} .
\end{aligned}
$$

Integration over $B$ brings one back to the initial expression for Wilson loop Eq. (38). We may choose, however, to integrate first over $\phi$. In that case we obtain an effective action for the massive $B$ field,

$$
W(C)=\int D B \exp \left[\int d x\left(\frac{16 \pi^{2}}{g^{2}} B_{\mu \nu}^{2}+f(H)\right)\right] \exp i \int_{\Sigma_{C}} B d \sigma,
$$

where $H=d B$ is the field strength and the function $H$ is given by (for details see Ref. 14)

$$
f(H)=\left[H \arcsin \left(\frac{H}{M^{2}}\right)\right]-\sqrt{M^{4}-H^{2}} .
$$

The multivaluedness of this action reflects the periodicity in $\phi$ mentioned ear-

lier. Expanding in powers of $B$ and limiting ourselves to quadratic terms, the effective action takes the form,

$$
S(B)=\frac{16 \pi^{2}}{g^{2}} \int d^{3} x\left[B_{\mu \nu}^{2}+\frac{\text { const }}{M^{2}} H_{\mu \nu \rho}^{2}\right] .
$$

For large loops one gets

$$
W(C) \sim \exp (-F(C, S)))
$$

with the following local expansion for the string action $F$ :

$$
F=c_{1} g^{2} M \int d^{2} \xi \sqrt{g}+c_{2} g^{2} M^{-1} \int d^{2} \xi\left(\nabla t_{\mu \nu}\right)^{2} \sqrt{g}+\ldots
$$

where

$$
g_{a b}=\partial_{a} \vec{x} \partial_{b} \vec{x}, \quad t_{\mu \nu}=\epsilon^{a b} \partial_{a} x_{\mu} \partial_{b} x_{\nu} .
$$


This is the action of the rigid string which also can be written as (see Ref. 17 and references therein)

$$
F=\int d^{2} \xi \sqrt{g} g^{a b} D_{a} \vec{x}\left(\sigma-s D^{2}+. .\right) D_{b} \vec{x}
$$

where $g$ and $D_{a}$ respectively are the determinant and the covariant derivative with respect to the induced metric $g_{a b}=\partial_{a} \vec{x} \partial_{b} \vec{x}$ on the embedded world sheet $\vec{x}\left(\xi_{1}, \xi_{2}\right)$. Note that the rigidity is controlled by the second term and one can show that the stiffness $s$ is parametrically of order

$$
s \propto \frac{\sigma}{M^{2}} .
$$

The stiffness $s$ in this expression is actually negative, but the system is stable due to higher order terms. Note that since $\sigma \propto g^{2} M \gg M^{2}$, the rigidity is very large, and is effective on the distance scales much larger parametrically than the "natural" string scale given by the string tension.

The action (49) explicitly depends on the surface $\Sigma$, while originally it was introduced as an unphysical object. The reason for this is the multivaluedness of the full action $S(B)$. As was shown in Ref. 14 the surface independence of $\langle W(C)\rangle$ is restored after summation over all possible surfaces.

\subsection{The confining string for pedestrians}

Let us now derive the action of a fluctuating string in a More direct way 19 We work with the Polyakov effective Lagrangian (14). Since the string world sheet is identified with the domain wall in the effective action, we will integrate in the partition function over all degrees of freedom apart from those that mark the position of the domain wall. To do this let us split the field $\phi$ into a continuous and discrete part

$$
\phi(x)=\hat{\phi}(x)+2 \pi \eta(x)
$$

where the field $\hat{\phi}$ is continuous but bounded within the vicinity of one "vacuum"

$$
-\pi<\hat{\phi}(x)<\pi
$$

and the field $\eta$ is integer valued. Clearly, whenever $\eta(x) \neq 0$, the field $\phi$ is necessarily not in the vicinity of the trivial vacuum $\phi=0$. Thus, the points in space where $\eta$ does not vanish mark in a very real sense the location of a domain wall between two adjacent vacua. The partition function $Z$ is given by the path integral

$$
\int \mathcal{D} \hat{\phi} \mathcal{D} \eta \quad \exp \left(-\frac{g^{2}}{16 \pi^{2}} \int d^{3} x\left[\frac{1}{2} \partial_{\mu}(\hat{\phi}+2 \pi \eta) \partial^{\mu}(\hat{\phi}+2 \pi \eta)\right.\right.
$$




$$
\left.\left.+M_{\gamma}^{2}(1-\cos \hat{\phi})\right]\right) .
$$

At weak coupling the field $\hat{\phi}$ can be integrated out in the steepest descent approximation. This means that the equations of motion for $\hat{\phi}$ have to be solved in the presence of the external source $\eta$, keeping in mind that $\hat{\phi}$ only takes values between $-\pi$ and $\pi$. Let us assume for this solution that the surfaces along which $\eta$ does not vanish are few and far between. Also, we will limit the possible values of $\eta$ to $0,1,-1$ corresponding to the "dilute gas" approximation. In fact, as we will see below, this exhausts all physically allowed values of $\eta$. Then the qualitative structure of the solution for $\hat{\phi}$ is clear. In the bulk, where $\eta$ vanishes, the field $\hat{\phi}$ satisfies normal classical equations. When crossing a surface $S$ on which $\eta=1$, the field $\hat{\phi}$ must jump by $2 \pi$ in order to cancel the contribution of $\eta$ to the kinetic term, since otherwise the action is ultraviolet divergent. Thus, the solution is that of a "broken wall". Far from the surface the field $\hat{\phi}$ approaches its vacuum value $\hat{\phi}=0$. Approaching $S$ it increases to $\pi$, then it jumps to $-\pi$ on the other side of the surface, and again approaches zero far from the surface. The profile of the solution for smooth $S$ (in this case a plane) is depicted in Fig. 1. Since outside the surface the field $\hat{\phi}$ solves classical equations of motion, clearly the profile of $\hat{\phi}$ is precisely the profile of the domain wall we discussed above. As noted above, the purpose of the discontinuity across the surface $\mathrm{S}$ is to cancel the would be ultraviolet divergent contribution of $\eta$ to the kinetic term in the action. Thus, the action of our solution is precisely

$$
S[\eta]=\sigma S=\sigma \int d^{2} \tau \frac{\partial X_{\mu}}{\partial \tau^{\alpha}} \frac{\partial X_{\mu}}{\partial \tau_{\alpha}},
$$

where $\tau$ are the coordinates on the surface $S$, and $X_{\mu}$ are the coordinates in the three-dimensional space-time. This is precisely the Nambu-Gotto action of a free string.

Of course the confining strings in the Georgi-Glashow model are not free. The thickness of the region in Fig. 1 in which the field $\hat{\phi}$ is different from zero, is clearly of order of the inverse photon mass $M_{\gamma}^{-1}$. Thus, when two surfaces come within distances of this order they start to interact. In principle this interaction can be calculated by just finding the classical solution for $\hat{\phi}$ in this situation. Now however we want to discuss one particular property of the confining strings - their rigidity.

\subsection{The string is soft ... and therefore rigid!}

The Nambu-Gotto action we have derived in the previous subsection is of course only the long wavelength approximation to the action of the confining 


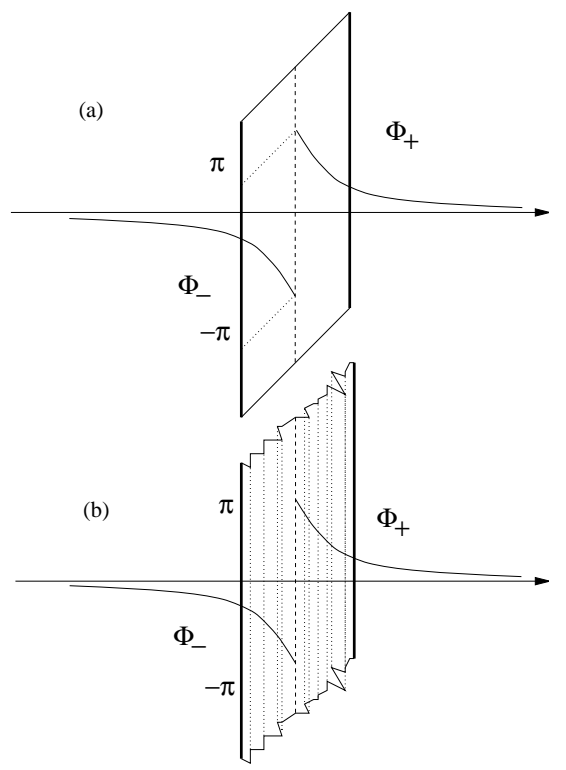

Figure 1: Schematic representation of the solution profile of the $\hat{\phi}$ field as a function of the $x$-coordinate. 


\section{$M W$}

Figure 2: The solid line represents the actual thin string. The dashed lines denote the contours of the effective thick string.

string. It is only valid for string world sheets which are smooth on the scale of the inverse photon mass. Expansion in powers of the derivatives can in principle be performed and it will give corrections to the action on scales comparable to $M_{\gamma}^{-1}$. However, for our confining string, strange things happen in the ultraviolet. Physically, the situation is quite peculiar, since the action of the string has absolutely no sensitivity to changes of the world sheet on short distance scales. This should be obvious from our derivation of the string action. Suppose that, rather than taking $\eta=1$ on an absolutely smooth surface, we make the surface look the same on the scale $M_{\gamma}^{-1}$ but add to it some wiggles on a much shorter distance scale $d$, as in Fig. 2. To calculate the action we now have to solve the classical equation for the field $\hat{\phi}$ with the new boundary condition - the surface of the discontinuity is wiggly. This new boundary condition changes the profile of the classical solution only within the thickness $d$ of the old surface. However, since the action of the classical solution is in no way dominated by the region of space close to the discontinuity, the action of the new solution will be the same as that of the old solution with the accuracy $d M_{\gamma}$. Thus, all string configurations which differ from each other only on small resolution scales, $d \ll M_{\gamma}^{-1}$, have to this accuracy the same energy! The string is therefore extremely soft, in the sense that it tolerates any number of wiggles on short distance scale without cost in energy, Fig. 3. In particular, since the string tension for our string is much greater than the square of the photon mass, $\sigma / M_{\gamma}^{2}=g^{2} / M_{\gamma} \gg 1$, fluctuations on the scale of the string tension are not penalized at all.

This independence of the action on short wavelength fluctuation is a dynamical manifestation of the so called zigzag symmetry introduced by Polyakov 14 Indeed, Polyakov notes that if a segment of a string goes back on itself, physically nothing has happened and so such a zigzag should not cost any action. 
This situation is an extreme example of a wiggle we have just discussed - a wiggle with infinite momentum. What happens physically is that not only do infinite momentum modes cost nothing, but so do finite but large momentum string modes.

The confining string is therefore very different from a weakly interacting string usually considered in the string theory. In the weakly interacting string, momentum modes with momentum of order of the square root of the string tension carry energy which is of the same order. In the confining string on the other hand, these momentum modes do not carry energy at all. Thus, we do not expect the spectrum of the confining string to contain states with large spatial momentum (heavy states with low angular momentum). In fact, the states with high angular momentum may very well be absent too, due to the fact that a long string can decay into a $W^{+}-W^{-}$pair. Therefore, it is likely that most of the string states will not appear in the spectrum of the Georgi-Glashow model. Thus, our confining string theory is quite peculiar: it is a string theory without stringy states in the spectrum.

In a somewhat perverse way, this softness of the "mathematical" string leads to rigidity of the physical string. The point is the following. As is obvious from the previous discussion, the high momentum modes are in a sense "gauge" degrees of freedom. The action of any string configuration does not depend on them. Consider a calculation of some physical quantity $O$ in the string path integral. If $O$ itself does not depend on the string high momentum modes, the integration over these modes factors out. If, on the other hand, $O$ does depend on them, then its average vanishes since their fluctuation in the path integral is completely random. This is absolutely analogous to the situation in gauge theories, where all observables must be gauge invariant, and for calculation of those the integration over the gauge modes always factors out, independently of the observable under consideration.

Thus, for all practical purposes, we should just exclude the high momentum string modes from consideration altogether. For example, when calculating the entropy of our string, we should only take into account the states which are different on the coarse grained level with the coarse graining scale of order $M_{\gamma}^{-1}$. This means that our string is intrinsically "thick". If we still want to describe this situation in terms of a mathematical string with the string tension $\sigma$, we must make the string rigid so that any bend on the scale smaller than $M_{\gamma}^{-1}$ is suppressed. Thus, such a string theory must have a curvature term with the coefficient of order $\sigma / M_{\gamma}^{2}$

$$
\delta S \propto \frac{\sigma}{M_{\gamma}^{2}}(\text { curvature) }
$$


This is indeed what happens in the truncated confining string action (49).

\subsection{The $W^{ \pm}$bosons - the 0 branes of the confining string}

Interestingly enough, the charged states of the Georgi-Glashow model have a natural place in the confining string framework. To represent a $W$ boson in the string language, let us consider a path integral Eq. (14) in the presence of one soliton. As we discussed above, within the effective Lagrangian (14) proper, solitonic configurations do not exist. However, they can be forced onto the system with the help of the external current. To create a winding state with world line $C$ we should insert a source in the path integral which forces a unit winding on the field $\chi$. In other words, the field $\phi$ would have to change by $4 \pi$ when going around $C$. The relevant partition function is

$$
Z[C]=\int D \phi \exp \left(-M_{W} L-S[\phi, j]\right)
$$

where

$$
S[\phi, j]=\frac{g^{2}}{16 \pi^{2}} \int d^{3} x\left[\frac{1}{2}\left(\partial_{\mu} \phi-2 \pi j_{\mu}\right)\left(\partial^{\mu} \phi-2 \pi j_{\mu}\right)+M_{\gamma}^{2}(1-\cos \phi)\right] .
$$

Here the "external current" $j_{\mu}$ is

$$
j_{\mu}(x)=n_{\mu}^{1}(x) \delta\left(x \in S_{1}\right)+n_{\mu}^{2}(x) \delta\left(x \in S_{2}\right)
$$

where $S_{1}$ and $S_{2}$ are two surfaces which both terminate on the curve $C$, and the unit vectors $n^{1}$ and $n^{2}$ are the normal vectors to these surfaces. The shape of the surfaces on which the current $j_{\mu}$ does not vanish is illustrated in Fig. 4. The insertion of $j_{\mu}$ forces the field $\phi$ to jump across the surface $S_{1}$ by $2 \pi$ and again jump by $2 \pi$ across $S_{2}$ in order to cancel the, otherwise ultraviolet divergent contribution of $j_{\mu}$. Thus, when going around $C$ the field $\phi$ changes by $4 \pi$ and therefore $C$ is the world line of the $W^{+}$boson. The position of the surfaces $S_{1}$ and $S_{2}$ is arbitrary as long as they both terminate on $C$. In particular, they could coincide, but here we prefer to use a more general form with non-coinciding surfaces.

As before, splitting the field variable $\phi$ into $\hat{\phi}$, and $\eta$ we see that the presence of $j_{\mu}$ just shifts the variable $\eta$ by unity on the two surfaces $S_{1}$ and $S_{2}$. The integration over $\hat{\phi}$ at fixed $\eta$ is performed in exactly the same way as before. The only difference now is that for any given $\eta$ one has two extra string world sheets along $S_{1}$ and $S_{2}$. After the integration over $\eta$, the result will not depend on the exact position of $S_{1}$ and $S_{2}$. However, at fixed $\eta$ the two surfaces 
introduced in Eq. (58) specify the positions of the two extra world sheets in the confining string path integral. We thus see that the field-theoretical path integral in the presence of a $W^{+}$boson, in the string representation, is given by the sum over surfaces in the presence of a $0-$ brane, which serves as a source for two extra string world sheets.

Let us stress here the similarity and differences between $W$ particles viewed as 0-branes, and D0 branes in string theory. Usually D0 branes are thought of as infinitely heavy. The situation in the Georgi-Glashow model is very similar in this respect. They are not infinitely heavy, but very heavy indeed since the mass of $W$ is large on all scales relevant to zero temperature physics. However, this analogy with D branes in superstring theory is not exact. The mass of a D0 brane is proportional to $1 / g_{s t r}$ whereas the mass of the $W$ boson (our 0 brane) is proportional to $g$. The fact that it is very heavy in the weak coupling limit is due to the large value of the Higgs condensate. It may be more natural to think of the $W$ as of a stretched F-string, which only after a duality transformation becomes a D-object on which the confining string can end. For the sake of brevity, in the following we will disregard this subtlety and will continue to refer to the $W$ bosons as 0 branes.

The contribution of the 0 brane to the partition function is suppressed by a very small factor $\exp \left\{-M_{W} L\right\}$, and vanishes for an infinitely large system. As we will see in the later sections however, the situation changes dramatically at finite temperature, where one dimension of the system has finite extent.

Incidentally, going back to our definition of the vortex field $V$, we see that in between the two world sheets the value of $V$ is negative, while outside it is positive. Thus, we have created a domain of the second vacuum of $V$ in between $S_{1}$ and $S_{2}$. It may be easier to visualize the situation with both $W^{+}$ and $W^{-}$present. In this case the surfaces $S_{1}$ and $S_{2}$ terminate on one side on the world line of $W^{+}$, and on the other side on the world line of $W^{-}$(see Fig. 5). They are thus boundaries of a closed domain of the second vacuum of the field $V$. In the infrared therefore our strings are nothing but the Ising domain walls, and the pair of D0 branes creates an Ising domain.

Note that in physical terms there are only two distinct vacua in the model: $\langle V\rangle=1$ and $\langle V\rangle=-1$. Thus, having two domain walls is the same as having a wall and an anti-wall, and if they coincide spatially such a configuration is equivalent to the vacuum. A configuration of $\eta$ with $\eta=2$ on a closed surface is physically equivalent to vacuum. Therefore, the values that $\eta$ is allowed to take are limited to 0,1 and -1 .

We close this section by noting, that the reason we have two string world sheets terminating on a D0 brane is that the field theory in question only has fields in the adjoint representation of $\mathrm{SU}(2)$. We can imagine adding 
heavy fundamental particles to the model. We would then also have allowed configurations of one string world sheet terminating on a D0 brane. These D0 branes would however be physically different and would have a different mass and therefore a different weight in the path integral. In field-theoretic terms, presence of the fundamental charges drastically changes the properties of the $Z_{2}$ magnetic symmetry, turning it into a local, rather than a global, symmetryed

\section{The Hamiltonian approach to compact $\mathbf{U}(1)$}

Our discussion so far has been in the context of the Lagrangian path integral formalism. Often, Hamiltonian approaches tend to be useful and more instructive. In this section we will therefore show how the same confinement phenomenon manifests itself in the canonical Hamiltonian analysis.

To make life a little easier in this section we will not study the $\mathrm{SU}(2)$ Georgi-Glashow model, but rather compact U(1) theory. This theory is also confining, and at low energies is indistinguishable from the Georgi-Glashow model. Since we will not at this point ask questions about the charged sector of the theory, compact $\mathrm{U}(1)$ is perfectly adequate for our purposes. In this section we follow Refs. 21 and 22 .

We start therefore by setting up the Hamiltonian description of U(1) compact QED. The first question to be settled, is what is the Hilbert space of admissible states. Firstly, it is clear that the Gauss' law should be implemented, and thus all the physical states should satisfy

$$
\exp \left\{i \int d^{2} x \partial_{i} \lambda(x) E_{i}(x)\right\}|\Psi\rangle=|\Psi\rangle .
$$

There is a crucial difference between the Gauss' law in the compact theory and in the noncompact one. In the noncompact theory equation (59) should be satisfied only for regular functions $\lambda$. For example, the operator

$$
V(x)=\exp \left\{\frac{i}{g} \int d^{2} y \frac{\epsilon_{i j}(x-y)_{j}}{(x-y)^{2}} E_{i}(y)\right\}
$$

which has the form of (59) with the function $\lambda$ proportional to the planar angle $\theta$, i.e. $\lambda=\frac{1}{g} \theta(x)$, does not act trivially on physical states. In fact, this operator creates point-like magnetic vortices with magnetic flux $2 \pi / g$ just like Eq. (26). It therefore changes the physical state on which it is acting.

In the compact theory the situation in this respect is quite different. Point like vortices with quantized magnetic flux $2 \pi n / g$ cannot be detected by any measurement. In the Euclidean path integral formalism of Ref. 1 this is the 
statement that the Dirac string of the monopole is unobservable and does not cost any (Euclidean) energy. In the Hamiltonian formalism this translates into the requirement that the creation operator of a point-like vortex must be indistinguishable from the unit operator. In other words, the operator (60) generates a transformation which belongs to the compact gauge group, and should therefore act trivially on all physical states. Equation (59) should therefore be satisfied also for these operators.

Accordingly, the Hamiltonian of the compact theory also must be invariant under these transformations. The magnetic field, defined as $B=\epsilon_{i j} \partial_{i} A_{j}$, on the other hand does not commute with $V(x)$ (cf. Ref. 23),

$$
V^{\dagger}(x) B(y) V(x)=B(y)+\frac{2 \pi}{g} \delta^{2}(x-y) .
$$

The Hamiltonian should therefore contain not $B^{2}$ but rather a periodic function of $B$. We will therefore choose our Hamiltonian to be

$$
H=\frac{1}{2} a^{2} \sum E_{\mathbf{n} i}^{2}-\frac{1}{g^{2} a^{2}} \sum \cos g a^{2} B_{\mathbf{n}} .
$$

Since we will need in the following an explicit ultraviolet regulator, we use lattice notations. Here $a$ is the lattice spacing, and the sums are respectively over the links and plaquettes of the two-dimensional spatial lattice. The coefficients of the two terms in the Hamiltonian are chosen so that in the weak coupling limit, upon formal expansion to lowest order in $g^{2}$, the Hamiltonian reduces to the standard free Hamiltonian of $2+1$ dimensional electrodynamics. Following Polyakov's book we work in the weakly coupled regime. Since the coupling constant $g^{2}$ in $2+1$ dimensions has dimension of mass, weak coupling means that the following dimensionless ratio is small

$$
g^{2} a \ll 1 .
$$

Our aim now is to find the vacuum wave functional of this theory. Exact solution of this problem is not feasible. However, at weak coupling we can use the variational approximation.

\subsection{The variational ansatz}

For a weakly coupled theory one expects the vacuum wave functional (VWF)

to be not too different from the vacuum of a free theory. Since the VWF of free (noncompact) electrodynamics is Gaussian in the field basis,

$$
\psi_{0}[\mathbf{A}]=\exp \left[-\frac{1}{2} \sum_{\mathbf{r}, \mathbf{s}} A_{\mathbf{r} i} G^{-1}(\mathbf{r}-\mathbf{s}) A_{\mathbf{s} i}\right],
$$


the Gaussian variational approach in this case should give a good approximation. An important caveat, however is that the ground state WF should be gauge invariant under the full compact gauge group. As a result it turns out that one can not take just a Gaussian in $\mathbf{A}$, since this will not preserve gauge invariance. The simplest generalization of the Gaussian ansatz which we use along the lines of Ref. 21, is to project a Gaussian WF into the gauge invariant subspace of the Hilbert space. The projection has to be performed with respect to the full compact gauge group of Eq. (59).

To facilitate this, define a vortex field $A_{\mathbf{n} i}^{V}$ that satisfies (we suppress the lattice spacing $a$ henceforth)

$$
\left(\nabla \times \mathbf{A}^{V}\right)_{\mathbf{n}^{\prime}}=\frac{2 \pi}{g} \delta_{b n^{\prime}, 0}, \quad \nabla \cdot \mathbf{A}^{V}=0
$$

where $\mathbf{n}^{\prime}$ is a plaquette on the lattice, or, equivalently, a site of the dual lattice. This is the vector potential corresponding to a magnetic field that is zero everywhere except at $\mathbf{n}^{\prime}=0$, where it takes the value $\frac{2 \pi}{g}$. The explicit solution of $(65)$ is

$$
A_{i}^{V}(\mathbf{n})=-\frac{2 \pi}{g} \epsilon_{i j}\left(\frac{\partial_{j}}{\nabla^{2}} \delta_{\mathbf{n}^{\prime}, 0}\right)_{\mathbf{n}}
$$

The compact gauge invariance requires that the variational wave function $\psi[\mathbf{A}]$ be invariant under shifts $\mathbf{A} \rightarrow \mathbf{A}+\mathbf{A}^{V}$. This of course is consistent with the periodicity of $H$ under $B \rightarrow B+\frac{2 \pi}{g}$. We also demand noncompact gauge invariance of the wave function. Hence we define a field, shifted by a noncompact gauge transformation $\phi_{\mathbf{n}}$ and by a vortex distribution $m_{\mathbf{n}^{\prime}}$,

$$
\mathbf{A}_{\mathbf{n}}^{(\phi, m)}=\mathbf{A}_{\mathbf{n}}-(\nabla \phi)_{\mathbf{n}}-\sum_{\mathbf{n}^{\prime}} m_{\mathbf{n}^{\prime}} \mathbf{A}^{V}\left(\mathbf{n}-\mathbf{n}^{\prime}\right)
$$

or, for short,

$$
A^{(\phi, m)}=A-\nabla \phi-A^{V} \cdot m .
$$

We choose the gauge invariant and periodic trial wave function as

$$
\psi[\mathbf{A}]=\sum_{\left\{m_{\mathbf{n}^{\prime}}\right\}} \int\left[d \phi_{\mathbf{n}}\right] \exp \left[-\frac{1}{2} \sum_{\mathbf{r}, \mathbf{s}} A_{\mathbf{r} i}^{(\phi, m)} G^{-1}(\mathbf{r}-\mathbf{s}) A_{\mathbf{s} i}^{(\phi, m)}\right] .
$$

Under a gauge transformation,

$$
\psi[\mathbf{A}+\nabla \lambda]=\psi[\mathbf{A}]
$$


since $\lambda$ can be absorbed in a shift in $\phi$. The simple rotational structure of $G_{i j}=\delta_{i j} G$ that appears in the variational wave function (69) is consistent with perturbation theory. We also take $G(x)$ to be a real function.

Our task now is to calculate the expectation value of the Hamiltonian in this state, and to minimize it with respect to the variational function $G$. We start by considering the normalization of the wave function.

\subsection{Normalization integral}

The normalization of $|\psi\rangle$ is

$$
\begin{aligned}
Z & \equiv\langle\psi \mid \psi\rangle=\sum_{\left\{m, m^{\prime}\right\}} \int[d \phi]\left[d \phi^{\prime}\right][d \mathbf{A}] \\
& \times \exp \left[-\frac{1}{2} A^{(\phi, m)} G^{-1} A^{(\phi, m)}\right] \exp \left[-\frac{1}{2} A^{\left(\phi^{\prime}, m^{\prime}\right)} G^{-1} A^{\left(\phi^{\prime}, m^{\prime}\right)}\right] .
\end{aligned}
$$

We shift $\mathbf{A}$ by $\nabla \phi^{\prime}+\mathbf{A}^{V} \cdot m^{\prime}$, and absorb the shift into $\phi$ and $m$, giving

$$
Z=\sum_{\{m\}} \int[d \phi][d \mathbf{A}] e^{-\frac{1}{2} A^{(\phi, m)} G^{-1} A^{(\phi, m)}} e^{-\frac{1}{2} A G^{-1} A} .
$$

Now we combine the exponents according to

$$
\begin{aligned}
A^{(\phi, m)} G^{-1} A^{(\phi, m)}+A G^{-1} A & =2 A^{(\phi / 2, m / 2)} G^{-1} A^{(\phi / 2, m / 2)} \\
& +\frac{1}{2} S(\phi, m) G^{-1} S(\phi, m),
\end{aligned}
$$

where

$$
\mathbf{S} \equiv \nabla \phi+\mathbf{A}^{V} \cdot m
$$

The first term in $\mathbf{S}$ has zero curl while the second is divergenceless. Furthermore, $G^{-1}$ is translation invariant and proportional to the unit matrix. Thus, $S G^{-1} S$ has no cross terms between $m$ and $\phi$. We now shift $\mathbf{A}$ by $\nabla \phi / 2+\mathbf{A}^{V} \cdot m / 2$, and all the fields decouple. We have then

$$
Z=Z_{A} Z_{\phi} Z_{v}
$$

where

$$
\begin{gathered}
Z_{A}=\operatorname{det} \pi G \\
Z_{\phi}=\int[d \phi] e^{-\frac{1}{4} \nabla \phi \cdot G^{-1} \cdot \nabla \phi}
\end{gathered}
$$




$$
\begin{gathered}
=\left(\operatorname{det} 4 \pi \frac{1}{\nabla^{2}} G\right)^{1 / 2}, \\
Z_{v}=\sum_{\left\{m_{\mathbf{n}^{\prime}}\right\}} \exp \left[-\frac{1}{4 g^{2}} \sum_{\mathbf{r}^{\prime}, \mathbf{s}^{\prime}} m_{\mathbf{r}^{\prime}} D\left(\mathbf{r}^{\prime}-\mathbf{s}^{\prime}\right) m_{\mathbf{s}^{\prime}}\right] .
\end{gathered}
$$

Here $Z_{v}$ is the vortex partition function, with the vortex-vortex interaction $D$ given by

$$
D\left(\mathbf{r}^{\prime}-\mathbf{s}^{\prime}\right)=g^{2} \sum_{\mathbf{r}, \mathbf{s}} \mathbf{A}^{V}\left(\mathbf{r}-\mathbf{r}^{\prime}\right) \cdot G^{-1}(\mathbf{r}-\mathbf{s}) \mathbf{A}^{V}\left(\mathbf{s}-\mathbf{s}^{\prime}\right),
$$

or

$$
D=-g^{2} A^{V} G^{-1} A^{V}=-4 \pi^{2} \frac{1}{\nabla^{2}} G^{-1} .
$$

We can split off the $\mathbf{r}^{\prime}=\mathbf{s}^{\prime}$ terms in (78) and write

$$
Z_{v}=\sum_{\left\{m_{\mathbf{n}^{\prime}}\right\}} \exp \left[-\frac{1}{4 g^{2}} \sum_{\mathbf{r}^{\prime} \neq \mathbf{s}^{\prime}} m_{\mathbf{r}^{\prime}} D\left(\mathbf{r}^{\prime}-\mathbf{s}^{\prime}\right) m_{\mathbf{s}^{\prime}}\right] \prod_{\mathbf{r}^{\prime}} z^{m_{\mathbf{r}^{\prime}}^{2}}
$$

where we have defined the vortex fugacity

$$
z=e^{-\frac{1}{4 g^{2}} D(0)} .
$$

In the interest of clarity, we adopt henceforth a continuum notation, indicating the ultraviolet cutoff $a$ only where necessary. Moreover, since the interesting physics comes from the infrared, lattice effects can be approximated by a momentum-space cutoff $\Lambda=a^{-1}$, which can simplify formulas further.

The variational function $G$ appears both in the vortex-vortex potential and in the vortex fugacity. We expect the ultraviolet behavior of $G$ to be the same as in the free theory, viz.,

$$
G^{-1}(k) \sim k
$$

so

$$
\begin{aligned}
D(0) & \sim \int^{\Lambda} \frac{d^{2} k}{(2 \pi)^{2}} \frac{4 \pi^{2}}{k^{2}} G^{-1}(k) \\
& \sim 2 \pi \Lambda
\end{aligned}
$$

and thus

$$
z \sim e^{-\frac{\pi}{2} \frac{1}{g^{2} a}}
$$

In the weak coupling region we have $z \ll 1$, justifying a restriction to $m=0, \pm 1$ in $(78)$ and (81). 


\subsection{Expectation values}

We calculate correlation functions of $m$ via a duality transformation 6 We add an $i J \cdot m$ term to the exponent in (78) and use the formula

$$
e^{-\frac{1}{4 g^{2}} m \cdot D \cdot m}=\mathrm{const} \int[d \chi] e^{-g^{2} \chi \cdot D^{-1} \cdot \chi} e^{i \chi \cdot m}
$$

to obtain

$$
Z_{v}=\int[d \chi] e^{-g^{2} \chi \cdot D^{-1} \cdot \chi} \prod_{\mathbf{n}}\left[1+2 \cos \left(\chi_{\mathbf{n}}+J_{\mathbf{n}}\right)\right] .
$$

Noting that ${ }^{a}$

$$
\cos (\chi+J)=\langle\cos \chi\rangle_{0}: \cos (\chi+J):=z: \cos (\chi+J):
$$

we have

$$
\begin{aligned}
Z_{v} & =\int[d \chi] e^{-g^{2} \chi \cdot D^{-1} \cdot \chi} \prod[1+2 z: \cos (\chi+J):] \\
& \simeq \int D \chi \exp \left[-g^{2} \chi D^{-1} \chi+2 z \int d^{2} x: \cos (\chi(x)+J(x)):\right]
\end{aligned}
$$

in continuum notation. Correspondingly 12

$$
\langle m(x) m(y)\rangle=2 g^{2} D^{-1}(x-y)-4 g^{4}\left\langle D^{-1} \chi(x) D^{-1} \chi(y)\right\rangle .
$$

The propagator of $\chi$ is easily calculated. To first order in $z$, the only contribution comes from the tadpole diagrams, which have already been subtracted in 90). Therefore,

$$
\begin{aligned}
\int d^{2} x e^{i k x}\langle\chi(x) \chi(0)\rangle & =\frac{1}{2 g^{2} D^{-1}(k)+2 z} \\
& =\frac{D(k)}{2 g^{2}}-z \frac{D^{2}(k)}{2 g^{4}}+O\left(z^{2}\right) .
\end{aligned}
$$

The correlation function of the vortex density is then

$$
K(k) \equiv \int d^{2} x e^{i k x}\langle m(x) m(0)\rangle=2 z+O\left(z^{2}\right),
$$

which in this approximation does not depend on momentum.

${ }^{a}$ The normal ordering is performed relative to the free theory defined by the quadratic action in 88 . 
Now we are ready to calculate the expectation value of the Hamiltonian (62). Using the definition (69) we obtain

$$
\begin{aligned}
& V^{-1}\left\langle\int E^{2} d^{2} x\right\rangle=-\frac{1}{V}\left\langle\psi\left|\sum_{\mathbf{n}, i} \frac{\partial^{2}}{\partial A_{\mathbf{n}, i}^{2}}\right| \psi\right\rangle \\
& =\frac{1}{2} \int \frac{d^{2} k}{(2 \pi)^{2}} G^{-1}(k)-\frac{\pi^{2}}{g^{2}} \int \frac{d^{2} k}{(2 \pi)^{2}} k^{-2} G^{-2}(k) K(k) \\
& =\frac{1}{2} \int \frac{d^{2} k}{(2 \pi)^{2}} G^{-1}(k)-\frac{2 \pi^{2}}{g^{2}} z \int \frac{d^{2} k}{(2 \pi)^{2}} k^{-2} G^{-2}(k)
\end{aligned}
$$

The magnetic part is easily calculated since it has an exponential form and, therefore, with our trial wave function leads to a simple Gaussian integral. We find

$$
\left\langle e^{i n g B_{\mathbf{n}}}\right\rangle=\exp \left[-\frac{1}{4} n^{2} g^{2} \int \frac{d^{2} k}{(2 \pi)^{2}} k^{2} G(k)\right]\left\langle e^{i n \pi m_{\mathbf{n}}}\right\rangle .
$$

The second factor is due to the vortices, and is different from unity only for odd values of $n$. Using (90) we find easily that $\left\langle e^{i \pi m}\right\rangle=e^{-4 z}$. Expanding to leading order in $g^{2}$ and $z$, we get

$$
\left\langle-\frac{1}{g^{2}} \cos g B\right\rangle=\frac{1}{4} \int \frac{d^{2} k}{(2 \pi)^{2}} k^{2} G(k)+\frac{4}{g^{2}} z,
$$

where we have dropped an additive constant. Finally, the expression for the variational vacuum expectation value of the energy is

$$
\frac{1}{V}\langle H\rangle=\frac{1}{4} \int \frac{d^{2} k}{(2 \pi)^{2}}\left[G^{-1}(k)+k^{2} G(k)-\frac{4 \pi^{2}}{g^{2}} z\left(k^{-2} G^{-2}(k)-\frac{4}{\pi^{2}}\right)\right] .
$$

\subsection{Determination of the ground state}

The expression (98) has to be minimized with respect to $G$. From equation (82) and (84) we find

$$
\frac{\delta z}{\delta G(k)}=\frac{1}{4 g^{2}} k^{-2} G^{-2}(k) z
$$


The variation of $(98)$ gives

$$
k^{2}-G^{-2}(k)=\frac{4 \pi^{4}}{g^{4}} z k^{-2} G^{-2}(k) \int \frac{d^{2} p}{(2 \pi)^{2}}\left[p^{-2} G^{-2}(p)-\frac{4}{\pi^{2}}\right] .
$$

Equation (100) has the solution

$$
G^{-2}(k)=\frac{k^{4}}{k^{2}+m^{2}}
$$

where

$$
m^{2}=\frac{4 \pi^{4}}{g^{4}} z \int \frac{d^{2} k}{(2 \pi)^{2}}\left[k^{-2} G^{-2}(k)-\frac{4}{\pi^{2}}\right] .
$$

The main contribution to the integral in the gap equation 102 comes from momenta $k^{2} \gg m^{2}$. For these momenta $k^{2} G^{-2}(k)=1$. Therefore, we see that (102) has a nontrivial solution. Using Eqs. (82), (84), and (101) we obtain

$$
m^{2}=\frac{4 \pi^{4}}{g^{4}} \exp \left(-\frac{\pi^{2}}{g^{2}} \int \frac{d^{2} p}{(2 \pi)^{2}} \frac{1}{\sqrt{p^{2}+m^{2}}}\right) \int \frac{d^{2} k}{(2 \pi)^{2}}\left[\frac{k^{2}}{k^{2}+m^{2}}-\frac{4}{\pi^{2}}\right]
$$

which for $g^{2} a=g^{2} / \Lambda \ll 1$ can be simplified to [cf. (86)]

$$
m^{2}=4 \pi^{2} \frac{\left(\pi^{2}-4\right) \Lambda^{4}}{g^{4}} \exp \left(-\frac{\pi \Lambda}{2 g^{2}}\right)
$$

where we have restored the ultraviolet cutoff dependence explicitly. The resulting $m$ is the mass gap of the theory, in the sense that it is the inverse of the spatial correlation length. Calculating, for example, the propagator of magnetic field, we find

$$
\left\langle e^{i g B_{\mathbf{m}}} e^{-i g B_{\mathbf{n}}}\right\rangle=\left|\left\langle e^{i g B}\right\rangle\right|^{2} e^{\frac{g^{2}}{2} \nabla^{2} G(\mathbf{m}-\mathbf{n})},
$$

and at large distances (neglecting power-like prefactors),

$$
\nabla^{2} G(x)=-\int \frac{d^{2} k}{(2 \pi)^{2}}\left(k^{2}+m^{2}\right)^{1 / 2} e^{i \mathbf{k} \cdot \mathbf{x}} \sim e^{-m x} .
$$

This dynamically generated mass is Polyakov's result Thus, we recover in the Hamiltonian approach the first important result - a finite mass gap $m$.

${ }^{b}$ We have dropped a term $-\frac{8 \pi^{2}}{g^{2}} z k^{-2} G^{-3}(k)$ from the right-hand side of 100 is smaller by a factor of $\frac{g^{2} k}{\Lambda^{2}}$ than the term retained, assuming $G \sim k^{-1}$ at large $k$. 
Let us note that a mass gap $m$ in a Hamiltonian formalism (104) has a different preexponential factor in comparison with a mass gap $M_{\gamma}$ obtained in a path integral approach (15). The reason is very simple. Contrary to universal exponential factor the preexponential factor depends on details of UV regularizations which are different in Lagrangian and hamiltonian formalisms.

\subsection{Spatial Wilson loops}

We also want to see whether the charges are confined in our best variational state. The simplest quantity that is related to confinement is the expectation value of the Wilson loop. Therefore, we will calculate it in our ground state,

$$
W_{l}[C]=\left\langle\exp \left(i l g \oint_{C} \mathbf{A} \cdot d \mathbf{x}\right)\right\rangle=\left\langle\exp \left(i l g \int_{\Sigma} B d S\right)\right\rangle,
$$

where $l$ is an integer and the integral is over the area $\Sigma$ bounded by the loop $C$. We have

$$
W_{l}[C]=\left\langle\prod_{S} e^{i l \pi m_{\mathrm{n}}}\right\rangle Z_{A}^{-1} \int D \mathbf{A} \exp \left(-A G^{-1} A+i l g \int_{\Sigma} B d S\right)
$$

The second factor is a Gaussian integral, which gives the factor

$$
W_{A}=\exp \left(\frac{l^{2} g^{2}}{4} \int_{\Sigma} d^{2} x \int_{\Sigma} d^{2} y \nabla^{2} G(\mathbf{x}-\mathbf{y})\right) .
$$

In the limit of large $\Sigma$ the leading behavior of the exponent is

$$
-\frac{l^{2}}{4} g^{2} \Sigma \lim _{k \rightarrow 0} k^{2} G(k)=-\frac{l^{2}}{4} g^{2} m \Sigma .
$$

This gives the area law with the string tension 9

$$
\sigma=\frac{l^{2}}{4} g^{2} m
$$

The first factor in Eq. (108) is different from unity only for odd $l$. It can be easily calculated to be

$$
\begin{aligned}
W_{v} \equiv\left\langle\prod_{S} e^{i l \pi m_{\mathbf{n}}}\right\rangle & =\int D \chi \exp \left[-g^{2} \chi D^{-1} \chi\right. \\
& \left.+2 z \int d^{2} x: \cos (\chi(x)-\alpha(x)):\right],
\end{aligned}
$$

${ }^{c}$ As was shown in Ref. 13 the dependence on $l$ in this formula is incorrect. The correct result is $\sigma \propto l$ rather than $\sigma \propto l^{2}$. For multiply charged Wilson loops the nonlinearities of the compact theory are important and the Gaussian variational ansatz may be inadequate. 
where $\alpha(x)$ vanishes for $x$ outside the loop and is equal to $\pi$ for $x$ inside the loop. At weak coupling we expand around a classical minimum of the exponent. Recall that the inverse propagator $D^{-1}$ is nonzero at zero momentum Eq. (80). This dictates the leading order solution $\chi(x)=0$ and

$$
W_{v}=e^{-4 z \Sigma} .
$$

This is a sub-leading correction to the string tension (111), since $g^{2} m \sim e^{-\pi / 4 g^{2}}$ and $z \propto m^{2} \ll g^{2} m$.

The behavior of the spatial Wilson loop suggests that the theory is confining with the string tension related in the expected way to the dynamically generated scale, $\sigma \propto g^{2} m$. However, the spatial Wilson loop does not directly give the potential between external charges. Although in the Euclidean formulation there is no difference between spatial and time-like Wilson loops, in the Hamiltonian approach one should be more careful. In particular, the Hamiltonian formulation does not preserve the Lorentz symmetry explicitly. It is therefore important to calculate the potential between external charges directly. Our variational ansatz can be extended to calculate explicitly such a potential22

\subsection{Interaction potential between external charges}

In the charged sector the gauge invariance condition $(7 \mathrm{~d})$ is replaced by

$$
\psi[\mathbf{A}+\nabla \lambda]=\psi[\mathbf{A}] \exp \left(i g \sum_{\mathbf{n}} \rho_{\mathbf{n}} \lambda_{\mathbf{n}}\right),
$$

where $\rho_{\mathbf{n}}$ is a fixed, integer background charge distribution. To calculate the interaction potential between two charges we will later take it to be a wellseparated dipole. A wave function that satisfies (114) is

$$
\begin{aligned}
\psi[\mathbf{A}] & =\sum_{\left\{m_{\mathbf{n}^{\prime}}\right.} \int\left[d \phi_{\mathbf{n}}\right] \exp \left[-\frac{1}{2} \sum_{\mathbf{r}, \mathbf{s}} A_{\mathbf{r} i}^{(\phi, m)} G^{-1}(\mathbf{r}-\mathbf{s}) A_{\mathbf{s} i}^{(\phi, m)}\right] \\
& \times \exp \left(i g \sum_{\mathbf{n}} \rho_{\mathbf{n}} \phi_{\mathbf{n}}\right) .
\end{aligned}
$$

The shifted field in (115) is defined along the lines of (68),

$$
A^{(\phi, m)}=A-\nabla \phi-A^{S} \cdot m .
$$


but now it is necessary to define the vortex field $A^{S}$ in a singular gauge. Like $A^{V}$, the field $A^{S}$ satisfies

$$
\left(\nabla \times \mathbf{A}^{S}\right)_{\mathbf{n}^{\prime}}=\frac{2 \pi}{g} \delta_{b n^{\prime}, 0} .
$$

While $A^{V}$ is divergenceless we take for $A^{S}$ the solution where $A$ is non-zero on the links dual to a string extending from 0 (the plaquette of the vortex) to $x=+\infty$,

$$
\begin{aligned}
& A_{\mathbf{n} x}^{S}=0, \\
& A_{\mathbf{n} y}^{S}= \begin{cases}\frac{2 \pi}{g} & \text { for } n_{x}>0, n_{y}=0 \\
0 & \text { otherwise. }\end{cases}
\end{aligned}
$$

Our reason for using $A^{S}$ rather than $A^{V}$ in (115) is one of locality. A dipole can be created in the vacuum (69) by the string operator $\exp \left[i g \sum_{\mathbf{x}}^{\mathbf{y}} A(\mathbf{z})\right]$, which places sources at $\mathbf{x}$ and $\mathbf{y}$. This operator creates a string of electric flux taking integer values along the string, the most local way of preserving Gauss' law when creating a dipole. A shift of $\mathbf{A}$ by $A^{S}$ commutes with this string operator, but a shift by $A^{V}$ does not. Shifting by $A^{V}$ will create a non-local, transverse electric field with fractional flux in addition to the string. In this light it appears unavoidable that the introduction of dynamical charges will immediately lead to a nonlocal and non-Lorentz-invariant theory. For more on this point see Ref. 24.

Another way of looking at it is to note that the difference between $A^{V}$ and $A^{S}$ can be absorbed by a shift in the integration variable $\phi_{\mathbf{r}}$ by $\frac{1}{g} \theta\left(\mathbf{r}-\mathbf{r}^{\prime}\right) m_{\mathbf{r}^{\prime}}$, where $\theta\left(\mathbf{r}-\mathbf{r}^{\prime}\right)$ is the angle that the vector $\mathbf{r}-\mathbf{r}^{\prime}$ makes with the $x$ axis. Using $A^{V}$ would therefore lead to an extra phase factor

$$
\exp \left[i \sum_{\mathbf{r}, \mathbf{r}^{\prime}} \rho_{\mathbf{r}} \theta\left(\mathbf{r}-\mathbf{r}^{\prime}\right) m_{\mathbf{r}^{\prime}}\right]
$$

in the integral in (115). Under a shift of vortex density $m$ this function is not invariant, but rather acquires a phase proportional to the charge density. The shift of vortex density can be viewed as a kind of large gauge transformation 21 and therefore this wave function belongs to a sector of the Hilbert space with a position-dependent " $\theta$ angle." It is hard to imagine how this sector can define a local theory, especially in the presence of dynamical charges.

Since $A^{S}$ differs from $A^{V}$ by a gauge transformation, it can be used interchangeably with it in the vacuum wave function (69). The distinction is 
only meaningful in the charged sector, where it leads to a new vortex-charge interaction (see (123) below).

The wave function (115) satisfies the Gauss' law, but it turns out to be a very poor variational state22 The reason is that it does not contain any extra variational parameter on top of the width of the Gaussian. Thus, the way the electric field, created by the external charges spreads in this state is not optimized. One can in fact calculate the distribution of the electric field22 This turns out to have a Coulomb-like profile. As a result, the energy of such a state is infrared divergent even for a finite size dipole. To do better we must introduce an extra variational parameter which could optimize the profile of the electric field. Thus, rather than using Eq. (115) we take as out state

$$
\begin{aligned}
\psi[\mathbf{A}]= & \sum_{\left\{m_{\mathbf{n}^{\prime}}\right\}} \int\left[d \phi_{\mathbf{n}}\right] \exp \left[-\frac{1}{2} \sum_{\mathbf{r}, \mathbf{s}} A_{\mathbf{r} i}^{(\phi, m)} G^{-1}(\mathbf{r}-\mathbf{s}) A_{\mathbf{s} i}^{(\phi, m)}\right] \\
& \times \exp \left(i g \sum_{\mathbf{n}} \rho_{\mathbf{n}} \phi_{\mathbf{n}}\right) \exp \left(i \sum_{\mathbf{n}} \mathbf{e}_{\mathbf{n}} \cdot \mathbf{A}_{\mathbf{n}}^{(\phi, m)}\right) .
\end{aligned}
$$

We take the classical background field $\mathbf{e}$ to be transverse, $\nabla \cdot \mathbf{e}=0$, and we will treat it as an additional variational parameter in the charged sector.

The normalization factor for the wave function (119) is

$$
Z=Z_{A} Z_{\phi}[\rho] Z_{v}[\rho]
$$

with

$$
\begin{aligned}
Z_{A} & =\operatorname{det} \pi G, \\
Z_{\phi}[\rho] & =\left(\operatorname{det} 4 \pi \frac{1}{\nabla^{2}} G\right)^{1 / 2} \exp \left(-g^{2} \rho \cdot \frac{1}{\nabla^{2}} G \cdot \rho\right), \\
Z_{v}[\rho] & =\sum_{\left\{m_{\mathbf{n}^{\prime}}\right\}} \exp \left[-\frac{1}{4 g^{2}} \sum_{\mathbf{r}^{\prime}, \mathbf{s}^{\prime}} m_{\mathbf{r}^{\prime}} D\left(\mathbf{r}^{\prime}-\mathbf{s}^{\prime}\right) m_{\mathbf{s}^{\prime}}\right] \\
& \times \exp \left(-i \sum_{\mathbf{r}^{\prime}} h_{\mathbf{r}^{\prime}} m_{\mathbf{r}^{\prime}}\right) .
\end{aligned}
$$

The new ingredient in (123) is a vortex-charge interaction,

$$
\sum_{\mathbf{r}^{\prime}} h_{\mathbf{r}^{\prime}} m_{\mathbf{r}^{\prime}} \equiv \sum_{\mathbf{r} \mathbf{r}^{\prime}}\left[\rho_{\mathbf{r}} \theta\left(\mathbf{r}-\mathbf{r}^{\prime}\right)+\frac{2 \pi}{g} \frac{1}{\nabla^{2}} \epsilon_{i j} \partial_{i}\left(\underline{\mathbf{r}}-\mathbf{r}^{\prime}\right) e_{j}(\mathbf{r})\right] m_{\mathbf{r}^{\prime}}
$$


The vortex interaction potential $D$ is again given by Eq. (80).

Let us now calculate the expectation value of the electric field in this state. Straightforward algebra gives

$$
\left\langle E_{i}\right\rangle=g \frac{\partial_{i}}{\nabla^{2}} \rho-\frac{i \pi}{g} G^{-1} \frac{\epsilon_{i j} \partial_{j}}{\nabla^{2}}\langle m\rangle+e_{i} .
$$

The energy of this state is

$$
\begin{aligned}
\langle H\rangle=\frac{V}{4} \int & \frac{d^{2} k}{(2 \pi)^{2}}\left[G^{-1}(k)+k^{2} G(k)\right]-\frac{g^{2}}{2} \int d^{2} x \rho \frac{1}{\nabla^{2}} \rho \\
& +\frac{\pi^{2}}{2 g^{2}} \int d^{2} x d^{2} y \partial^{-2} G^{-2}(x-y)\langle m(x) m(y)\rangle \\
& -\frac{1}{g^{2}} \int d^{2} x\left(\operatorname{Re}\left\langle e^{i \pi m(x)}\right\rangle-1\right) \\
& +\frac{i}{2} \zeta G^{-1}\langle m\rangle+\frac{1}{2} \int d^{2} x e^{2},
\end{aligned}
$$

where the penultimate term contains the potential $\zeta(x)$, defined via

$$
e_{i}=\frac{g}{2 \pi} \epsilon_{i j} \partial_{j} \zeta .
$$

To calculate correlation functions of the vorticity $m$, we again introduce the dual field $\chi$ as in Eq. (87) and obtain the relations

$$
\begin{aligned}
\langle m\rangle & =-2 i g^{2} D^{-1}\langle\chi\rangle, \\
\langle m(x) m(y)\rangle & =2 g^{2} D^{-1}-4 g^{4}\left\langle D^{-1} \chi(x) D^{-1} \chi(y)\right\rangle .
\end{aligned}
$$

The duality transformation now results in the following Lagrangian for the dual field $\chi$,

$$
\mathcal{L}=g^{2} \chi D^{-1} \chi-2 z \int d^{2} x: \cos (\chi(x)+\zeta-\theta \rho): .
$$

The notation $\theta \rho$ represents the convolution of the source distribution $\rho(\mathbf{r})$ with $\theta\left(\mathbf{r}-\mathbf{r}^{\prime}\right)$.

To first order in $z$ we obtain

$$
\begin{aligned}
\langle m(x)\rangle & =-2 i z \sin (\theta \rho(x)-\zeta(x)), \\
\langle m(x) m(y)\rangle & =2 z \cos (\theta \rho(x)-\zeta(x)) \delta^{2}(x-y), \\
\left\langle e^{i \pi m(x)}\right\rangle & =\exp [-4 z \cos (\theta \rho(x)-\zeta(x))] .
\end{aligned}
$$


The e-dependent piece of the energy is

$$
\begin{aligned}
\Delta E=\quad & m^{2} g^{2} \int d^{2} x[1-\cos (\theta \rho(x)-\zeta(x))] \\
& +z \int d^{2} x d^{2} y \zeta(x) G^{-1}(x-y) \sin (\theta \rho(y)-\zeta(y)) \\
& -\frac{g^{2}}{8 \pi^{2}} \int d^{2} x \zeta \nabla^{2} \zeta .
\end{aligned}
$$

The quantity (131) is to be minimized with respect to $\zeta$. It is obvious immediately that at large distances from the sources $\zeta \rightarrow \theta \rho$, so that the energy is infrared finite. Noting that the second term is of order $g^{2}$ relative to the first, we drop it from now on. The minimization equation for $\zeta$ then becomes very simple,

$$
\nabla^{2} \zeta-m^{2} \sin (\zeta-\theta \rho)=0
$$

To study Eq. (132) it is convenient to define $\tilde{\zeta}=\zeta-\theta \rho$ which satisfies the sine-Gordon equation with a singular source term,

$$
\nabla^{2} \tilde{\zeta}-m^{2} \sin \tilde{\zeta}=S
$$

The source term $S$ consists of a dipole layer along the line between the external point charges. For charges separated by a distance $L$, we have

$$
S(\mathbf{x})=2 \pi \delta^{\prime}\left(x_{2}\right) \eta\left(\frac{L}{2}+x_{1}\right) \eta\left(\frac{L}{2}-x_{1}\right)
$$

where $\eta$ is a step function. When $L$ is much larger than $1 / \mathrm{gm}$ the solution of Eq. 133 can be found in the region $-L / 2 \ll x_{1} \ll L / 2$. In this region $\tilde{\zeta}$ is approximately independent of $x_{1}$ and satisfies the one-dimensional sine-Gordon equation in $x_{2}$,

$$
\frac{\partial^{2} \tilde{\zeta}}{\partial x_{2}^{2}}-m^{2} \sin \tilde{\zeta}=S
$$

The singular source $S$ creates a discontinuity of $2 \pi$ at $x_{2}=0$. The solution for $x_{2}>0$ is hence half a sine-Gordon soliton, with $\tilde{\zeta}=\pi$ at $x_{2}=0+$ and $\tilde{\zeta} \rightarrow 0$ as $x_{2} \rightarrow \infty$. For $x_{2}<0$ it is half the anti-soliton with $\tilde{\zeta}=-\pi$ at $x_{2}=0-$ and $\tilde{\zeta} \rightarrow 0$ as $x_{2} \rightarrow-\infty$. At distances greater than $1 / m$ from the $x_{1}$ axis, $\tilde{\zeta}$ vanishes exponentially. Therefore, we have $\zeta \rightarrow \theta \rho$ exponentially. The vortex density $m(x)$ according to Eq. (130) vanishes exponentially. Referring to Eq. (125) we see that the total electric field vanishes exponentially outside the region of width $1 / m$, indeed forming a flux tube of thickness $1 / m$. This 
is perfectly in accord with our expectation for the field profile in a confining theory.

In Eq. (131) we see that the energy density of the flux tube is proportional to the energy of the sine-Gordon soliton solution with the proportionality coefficient $\frac{g^{2}}{4 \pi^{2}}$. The string tension is therefore

$$
\sigma=\frac{2}{\pi^{2}} g^{2} m .
$$

This is consistent with the result of the calculation of the spatial Wilson loop in the previous subsection. We have thus established that the electric field in the vacuum of compact QED is squeezed into flux tubes, and that the energy of a large dipole is proportional to its length.

\subsection{Relation between the Lagrangian and the Hamiltonian pictures}

Some words should be said about the relation of the two-dimensional Hamiltonian calculation to the three-dimensional Euclidean path integral. The vacuum wave functional of the theory can be represented in path integral formalism. To get the vacuum WF $\Psi[A]$ one should calculate the path integral over the fields $A(x, t)$, with $t$ varying from $-\infty$ to 0 , with the boundary condition $A(x, t=0)=A(x)$. To be more precise, in calculating the VEV of some operator $O(t=0)$, one should split the time coordinate of the plane with the time coordinate of the operator, so that one considers $\Psi[A(x, t=-\epsilon)]$ and $\Psi^{*}[A(x, t=\epsilon)]$ in the limit $\epsilon \rightarrow 0$.

The basic objects that appear in the Euclidean path integrals are monopoles, which in 3D are not propagating particles, but rather instantons. When described in terms of the vector potential, or noncompact field strength, a monopole has a Dirac string attached to it. It is clear that the vortices (antivortices) in Eq. (69) correspond precisely to the intersections of the Dirac strings of the 3D monopoles (anti-monopoles) with the equal time plane at $t=0$. The positions of the Dirac strings are not physical in the compact theory, and only the position of the monopole itself is gauge invariant. In fact, for all monopoles that do not sit in the infinitesimally thin time slab between the planes $t=-\epsilon$ and $t=\epsilon$, one can always choose the direction of the Dirac string such that it does not intersect the two planes. This precisely corresponds to the shift of the integration variable in going from Eq. (71) to Eq. (72). The combination that enters this path integral nontrivially is the difference in vorticity between $\Psi$ and $\Psi^{*}$. At points where both $m$ (which corresponds to $\Psi$ ) and $m^{\prime}$ (which corresponds to $\Psi^{*}$ ) are nonzero but equal, the difference vanishes. This is the situation when a Dirac string intersects both planes $t= \pm \epsilon$. 
When a 3D monopole sits in the slab, only one of $m$ or $m^{\prime}$ does not vanish and so their difference is nonzero. The summation over $m$ in Eq. (72) can be interpreted therefore as the direct contribution to the partition function due to the monopoles at precisely the time $t=0$.

The fact that in this way one sees directly only the monopoles at $t=0$, does not mean of course that other monopoles are not taken into account in this approximation. Indeed, the "bare" interaction potential between the $t=0$ monopoles is $D(x)$. In the best variational state it is already short range, as follows from the solution for $G(k)$ (see Eq. (101)). This is in accord with the $3 \mathrm{D}$ picture, where the $3 \mathrm{D}$ monopole gas produces screening. Obviously, if one only looks at the thin slab, every monopole there will have an anti-monopole partner, which sits nearby (inside the screening length) in the third direction. The 2D monopole gas will therefore be screened by the 3D interaction, even before the interactions of the 2D monopoles between themselves is taken into account. This is perfectly consistent with our calculation. It is interesting to note, that even though this $2 \mathrm{D}$ interaction produces additional screening (the cosine term in the effective theory (90)), it is the 3D screening that is responsible for the area law of the Wilson loop, as is clear from the calculation of the string tension in Eq. (108). In fact, if one takes the noncompact expression $G^{-1}(k)=|k|$, exen the subleading term in the area law in the Wilson loop (113) disappears 21

\section{The deconfining phase transition}

Now that we understand confinement in some detail, it is time to ask how it disappears. The deconfining phase transition in QCD at finite temperature has been the subject of numerous studies in recent years. Some aspects of the physics of the high temperature phase appear to be perturbative and can be studied in a controlled way at asymptotically high temperatures. However, in the phase transition region itself the QCD coupling is large, and the physics is dominated by the nonperturbative soft sector. Analytic understanding of this region is therefore extremely difficult. It is thus again useful to fall back on our toy model where a similar deconfining phase transition is within the weak coupling regime and can be studied analytically.

In this section we discuss in detail the physics of the deconfining phase transition. Analysis of the deconfining phase transition in the compact $\mathrm{U}(1)$ theory was performed in Ref. 25. The SU(2) Georgi-Glashow model was studied in Ref. 26. Interestingly enough, although at zero temperature the differences between these two models are minute, the behavior close to the phase transition is

very distinct. So much so that the dynamics that drives the phase transition as 
well as the universal characteristics of the transition are completely different. In particular, whereas in the compact $\mathrm{U}(1)$ theory the transition is apparently driven by the binding of monopoles, in the Georgi-Glashow model it is due to the plasma effects of charged excitations ( $W$ bosons). In the lattice framework the monopole binding in the compact $\mathrm{U}(1)$ theory has been recently studied in Ref. 27. One word of caution though, is that the compact $U(1)$ theory does not have a proper continuum limit. Thus, it is not entirely clear whether the monopole binding mechanism is relevant to continuum field theory. In this review therefore we will concentrate on the Georgi-Glashow model.

\subsection{The monopole binding}

Since at zero temperature the monopole contributions are the only relevant ones for confinement, one may be tempted to assume that also at finite, low enough temperature all other effects are unimportant. The expected value of the transition temperature, as we shall see in a moment, is of order $g^{2}$, which is much smaller than any mass scale in the theory except for the photon mass. One could therefore start with the working hypothesis that the single selfinteracting photon field (or, equivalently, the monopole ensemble) should be a valid description of the phase transition region. This scenario was discussed in Ref. 25.

The physics here is simple. The first thing to note is that at finite temperature the interaction between monopoles is logarithmic at large distances. The reason is that the finite temperature path integral is formulated with periodic boundary conditions in the Euclidean time direction. The field lines are therefore prevented from crossing the boundary in this direction. The magnetic field lines emanating from a monopole have to bend close to the boundary and go parallel to it. So effectively the whole magnetic flux is squeezed into two dimensions. Qualitatively the situation is shown in Fig. 3.

The length of the time direction is $\beta=1 / T$, and thus the field profile is clearly two-dimensional on distance scales larger than $\beta$. Two monopoles separated by a distance larger than $\beta$ therefore interact via a two-dimensional rather than a three-dimensional Coulomb potential, which is logarithmic at large distances. Since the density of the monopoles is tiny $\rho_{M} \propto \xi$, the monopole gas becomes two-dimensional already at extremely low temperatures $T \propto \xi^{1 / 2}$. The strength of the logarithmic interaction is easily calculated. The magnetic flux of the monopole should spread evenly in the compact direction once we are far enough from the monopole core. The field strength should have only components parallel to the spatial directions. Since the total flux of the monopole 


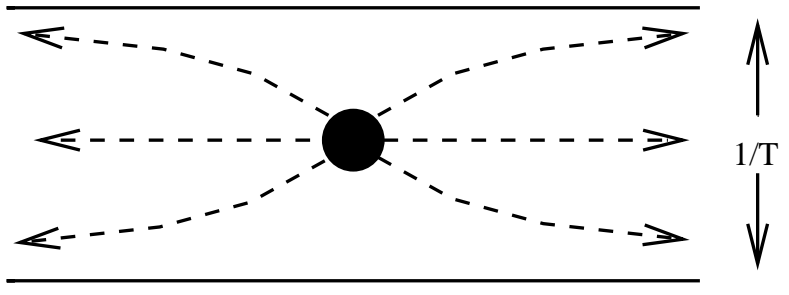

Figure 3: The field of a monopole-instanton at finite temperature.

is $2 \pi / g$, the field strength far from the core is

$$
\tilde{F}_{i}=\frac{T}{g} \frac{x_{i}}{x^{2}},
$$

and thus the strength of the infrared logarithmic interaction is $T^{2} / g^{2}$.

It is well known 28 that the two-dimensional Coulomb gas undergoes the Berezinsky-Kosterlitz-Thouless (BKT) phase transition. In the usual Coulomb gas, where the strength of the interaction $\lambda$ does not depend on temperature, the particles are bound in pairs at low temperature and unbind at high temperature $T>T_{\mathrm{BKT}}=2 \pi \lambda$, where the entropy overcomes the energy. This is the standard BKT phase transition 28 which determines the universality class of $\mathrm{U}(1)$ symmetry restoration in 2 dimensions. In the present case the situation is reversed, since the strength of the interaction itself depends on the temperature. At low temperature the interaction is weak, and therefore the particles (monopoles) are free. As the temperature grows, the interaction becomes stronger until at $T_{\mathrm{BKT}}=2 \pi T_{\mathrm{BKT}}^{2} / g^{2}$ the energy overcomes the entropy. Above this temperature, the monopoles bind into neutral pairs. Thus, based on this simple picture one can expect the theory to undergo a BKT phase transition at

$$
T_{\mathrm{BKT}}=\frac{g^{2}}{2 \pi} .
$$

Below this temperature the photon should be massive, while above this temperature it should be massless, since the cosine term in the Lagrangian (14) is irrelevant.

This is precisely the logic followed in Ref. 25. One can go further and perform more quantitative calculations using the dimensionally reduced version of Polyakov's effective Lagrangian (14). Dimensional reduction should be 
perfectly valid in this theory since the critical temperature is much larger than the photon mass. The effect of the monopoles at finite temperature is thus contained in the two-dimensional model

$$
\mathcal{L}=\frac{g^{2}}{32 \pi^{2} T}\left(\partial_{i} \phi\right)^{2}+\frac{M^{2} g^{2}}{16 \pi^{2} T} \cos \phi .
$$

This theory, in full agreement with the previous discussion has a BKT phase transition at the temperature given by Eq. (137), above which the "dual photon" field $\phi$ becomes massless.

Moreover, since the two-dimensional sine-Gordon theory is exactly solvable, one can calculate various correlation functions. In particular, the string tension was calculated in Ref. 25, and was found to exhibits quite strange behavior. At low temperatures it follows an expected pattern - that is, it decreases with temperature. It becomes extremely small at temperature much smaller than $T_{\mathrm{BKT}}$; however, just before $T_{\mathrm{BKT}}$ it starts rising sharply and at $T_{\mathrm{BKT}}$ actually diverges.

Although the logic leading to the previous discussion seems quite sound, there are several puzzles that arise regarding the results.

- The photon becomes massless in the high temperature phase. In other words, the correlation length in some physical (gauge invariant) channel becomes infinite at high temperature. This contradicts our physical intuition, since one expects that at high temperature any physical system described by a local field theory should become maximally disordered with zero correlation length.

- The phase transition is of BKT type, and therefore belongs to the U(1) universality class. On the other hand, we know 11 that the global symmetry that is restored at the phase transition is the magnetic $Z_{2}$, and we expect the universality class to be $2 \mathrm{D}$ Ising. This is also expected from the classic universality arguments 29

- The divergence of the string tension at the critical point looks unphysical.

- Finally, as we explained in the previous sections, the effective theory (14) does not allow charged states. It is then difficult to understand in what sense the high temperature phase can be viewed as deconfined.

\subsection{Magnetic symmetry restoration and the charged plasma}

Another line of argument starts with the realization that confinement is tantamount to the spontaneous breaking of the $Z_{2}$ magnetic symmetry. Indeed, let us now take Eq. (28) as our starting point. What does one expect from the phase transition in a simple scalar theory of this type? Firstly, clearly one 
expects the critical temperature to be of order of the expectation value of the scalar field, and therefore parametrically of order $g^{2}$. At finite temperature one expects the generation of a positive thermal mass proportional to the temperature and to the coupling constant. Thus, the thermal contribution to the effective potential should be of the form

$$
\delta_{T} \mathcal{L}=x \lambda T V^{*} V .
$$

At $T=g^{2} / 4 \pi^{2} x$ the total mass term becomes positive, the VEV of $V$ vanishes and the phase transition occurs. One can estimate this temperature more precisely using the following simple argument. Let us neglect for now the monopole induced term. Then we are dealing with the $X Y$ model at finite temperature. The dimensional reduction should again be a valid approximation, and thus essentially we have to analyze a 2D model. Now a 2D $X Y$ model can be mapped into a sine-Gordon theory of a dual field $\tilde{\chi}$. Performing this dual transformation 9 , we find the Lagrangian

$$
\mathcal{L}=\frac{T}{2 g^{2}}\left(\partial_{i} \tilde{\chi}\right)^{2}+\mu \cos \tilde{\chi}
$$

where $\mu$ is the fugacity of the vortices in the $X Y$ model. This sine-Gordon theory has a phase transition at

$$
T_{X Y}=\frac{g^{2}}{8 \pi} .
$$

Therefore, we may expect the magnetic symmetry restoring phase transition at $T_{X Y}$. Although parametrically this temperature is of the same order as the BKT transition temperature discussed in the previous subsection, it is four times lower. Another important difference is that the nature of the phase transition is in fact completely different. The phase transition in the model (140) is due to the unbinding of vortices of the field $V$. Above $T_{X Y}$ the vortices are in the plasma phase. These vortices are, as discussed earlier, precisely the charged $W^{ \pm}$bosons of the original Georgi-Glashow model. Thus, this phase transition is just what one would naturally call the deconfining phase transition and the vacuum above $T_{X Y}$ is the charged plasma.

Note that in this discussion we have neglected completely the effect of monopoles, that is the last term in the Lagrangian (28). Interestingly enough we see that the plasma phase is reached at a temperature which is much lower than $T_{\mathrm{BKT}}$ discussed earlier, and thus the monopole binding is irrelevant for

${ }^{d}$ To fix the normalization of the kinetic term we should bear in mind that a vortex corresponds to a $2 \pi$ jump in the field $\chi=2 \phi$ rather than the field $\phi$ in Eq. (14). 
the dynamics of the deconfinement. This is not to say that the presence of the monopoles is irrelevant altogether. Clearly, omitting the monopole induced term, we enlarged the symmetry of the system from $Z_{2}$ to $\mathrm{U}(1)$. Hence the effective $X Y$ model description and the $\mathrm{U}(1)$ universality class predicted by Eq. (140). The analysis as it stands is correct for noncompact electrodynamics with charged matter, but not for the Georgi-Glashow model. Next we remedy this problem.

The effect of the monopoles can be qualitatively understood in a simple way. Their presence leads to a confining potential between the charges, that is a linear interaction between the $X Y$ model vortices. Thus, the monopoles suppress the variation of the phase of the field $V$ except inside the confining string, the width of which is given by the inverse photon mass $d=1 / M \propto \xi^{1 / 2}$. Whether the presence of the monopoles is important at the would be phase transition point $T_{X Y}$ depends crucially on the density of charged particles. If the density of the charged particles is very high, so that the average distance between them is smaller than $d$, the presence of the monopoles is immaterial since the phase of $V$ is disordered already on the short distance scale. However, if the density of charges is low, so that the distance between them is larger than $d$, the presence of the monopoles will suppress the phase transition. In this case at $T_{X Y}$ the vacuum will not be disordered, but will look like a dilute gas of charged particles with strings between them. The actual phase transition will then occur at a higher temperature, where the average distance between the charges equals the inverse photon mass.

Interestingly enough in the present case this temperature turns out to be twice the value of $T_{X Y}$ and therefore still much lower than $T_{\mathrm{BKT}}$. We will show this rigorously in the next subsection. Qualitatively though this is easy to understand. The density of charges is proportional to their fugacity

$$
\mu \propto e^{\frac{-M_{W}}{T}} .
$$

This should be compared to the square of the photon mass, which is given by the zero temperature monopole fugacity. In a theory with very heavy Higgs

$$
\xi \propto e^{\frac{-4 \pi M_{W}}{g^{2}}} .
$$

The two are equal (up to subleading corrections) at

$$
T_{\mathrm{GG}}=\frac{g^{2}}{4 \pi} .
$$

More generally, when the Higgs is not infinitely heavy, the monopole fugacity 
is smaller and thus the transition temperature will be lower,

$$
T_{c}=\frac{g^{2}}{4 \pi \epsilon\left(\frac{M_{H}}{M_{W}}\right)} .
$$

For a lighter Higgs, $T_{c}$ gets closer to $T_{X Y}$, but is always greater, so that $T_{X Y}<T_{c}<T_{\mathrm{BKT}}$.

In the next subsection we will present a more complete analysis based on the renormalization group and exact bosonization. This analysis confirms the simple picture presented here. Thus, all the puzzles raised in the previous subsection simply disappear:

- The photon never becomes massless. Even without the monopoles in the plasma phase it acquires the Debye mass given by the cosine term in Eq. (140). This mass rises with temperature, and thus the physical correlation length decreases.

- Since the monopole term is still relevant at the phase transition, the universality class must be $Z_{2}$. We will see this explicitly in the next section.

- The analysis of the previous subsection is only valid below $T_{X Y}$, and thus the divergence of the string tension at $T_{\mathrm{BKT}}>T_{X Y}$ has nothing to do with the physics of the Georgi-Glashow model.

- Finally, the phase transition is driven primarily by the unbinding of charged particles and thus indeed has all the flavor of a deconfinement transition.

Let us now turn to a more quantitative analysis of what is stated above.

\subsection{The renormalization group analysis}

For a more formal analysis of the phase transition we find it convenient to use the sine-Gordon formulation in terms of the phase field $\chi$ modified to take into account explicitly the finite probability of the appearance of vortices. This Lagrangian has the form

$$
\mathcal{L}=\frac{g^{2}}{8 \pi^{2} T}\left(\partial_{\mu} \chi\right)^{2}+\zeta \cos 2 \chi+\mu \cos \tilde{\chi},
$$

where $\zeta$ is related to the monopole fugacity $\zeta=\xi / T$ and $\tilde{\chi}$ is the field dual to $\chi$,

$$
i \partial_{\mu} \tilde{\chi}=\frac{g^{2}}{2 \pi T} \epsilon_{\mu \nu} \partial^{\nu} \chi
$$


The way to derive this Lagrangian is as follows. The partition function of the sine-Gordon model in the presence of one vortex is

$$
Z(x)=\int D[\chi] \exp \left[-\int d^{2} y \frac{g^{2}}{8 \pi^{2} T}\left(\partial_{\mu} \chi-j_{\mu}(y, x)\right)^{2}+\zeta \cos 2 \chi\right] .
$$

The "external current" is

$$
j_{\mu}(y, x)=2 \pi n_{\mu}(y) \delta(y \in C)
$$

with $C$ a curve that starts at the location of the vortex (the point $x$ ), and goes to infinity, and $n_{\mu}$ is the unit normal to this curve. The insertion of this current forces the derivative of $\chi$ to have a discontinuity across the curve $C$, so that $\chi$ jumps by $2 \pi$. This forces $\chi$ to have one unit of vorticity concentrated at the point $x$. Note that even though $j_{\mu}$ explicitly depends on the curve $C$, the partition function itself does not, since changing the integration variable

$$
\chi(x) \rightarrow \chi(x)+2 \pi, \quad x \in S,
$$

where the boundary of $S$ is $C-C^{\prime}$, is equivalent to changing $C$ into $C^{\prime}$ in the definition of the current. The extra linear term in the exponential in Eq. (148) is

$$
i \tilde{\chi}=\frac{g^{2}}{2 \pi T} \int_{C} d x_{\mu} \epsilon_{\mu \nu} \partial_{\nu} \chi
$$

which is equivalent to Eq. (147). An anti-vortex at $y$ is obviously created by $-j_{\mu}$. To create several vortices one just inserts an external current which is the sum of the currents which create individual vortices.

A dilute ensemble of vortices and anti-vortices with (small) fugacity $\mu$ is then given by

$$
Z=\sum_{n, m} \frac{1}{n !} \frac{1}{m !} \mu^{n+m} \int \Pi_{i} d x_{i} \Pi_{j} d y_{j} Z\left(x_{i}, y_{j}\right)
$$

The summation over the number of vortices and anti-vortices can be easily performed leading to the partition function with the Lagrangian (146). The constant $\mu$ is the vortex fugacity scaled by the effective ultraviolet cutoff imposed on the integration over the coordinates. The vortex fugacity of course is none other but the fugacity of the charged $W$,

$$
\mu=a^{-2} e^{\frac{-M_{W}}{T}} .
$$

The cutoff $a$ is related to the Compton wavelength of the $W$ boson, but a more careful determination of it should take into account the fact that in the 
process of dimensional reduction, all modes with frequencies above $T$ have been integrated out. We will not attempt the determination of $a$, but only note that it is some combination of the scales $M_{W}$ and $T \propto g^{2}$, and as such its value always plays a role secondary to the exponential factor of fugacity.

An alternative way to derive the Lagrangian $(146)$ is to start directly from the effective theory (28) with the extra Skyrme term (35). In the nonlinear $\sigma$-model limit one can cleanly separate the phase of the field $V$ into a smooth part $\chi$ and the vortex contribution. The Skyrme term then is proportional to the energy of one vortex and, with the Skyrme coupling chosen the way we discussed in Sec. 2, is just equal to $M_{W}$ for a one vortex configuration. The dilute vortex gas approximation then reproduces the Lagrangian (146).

Since both $\xi$ and $\mu$ are small, the importance of different terms in the Lagrangian (146) is determined by their respective conformal dimensions calculated in the free theory. The total conformal dimensions of the operators $: \cos 2 \chi:$ and $: \cos \tilde{\chi}:$, respectively, are

$$
\Delta_{\xi}=\frac{4 \pi T}{g^{2}} \quad \text { and } \quad \Delta_{\mu}=\frac{g^{2}}{4 \pi T} .
$$

Thus, at low temperature the charges are irrelevant $\left(\Delta_{\mu}>2\right)$, the monopoles are relevant $\left(\Delta_{\xi}<2\right)$ and the theory reduces to the sine-Gordon model. At the temperature $T=g^{2} / 4 \pi$ the two operators become equally relevant, since their conformal dimensions are equal,

$$
\Delta_{\mu}=\Delta_{\xi}=1 .
$$

The phase transition point can be determined from the structure of the fixed points of the renormalization group equations. The critical point is the infrared unstable fixed point of the renormalization group flow. The renormalization group equations of the sine-Gordon theory have been studied perturbatively in the literature both in the absence 6 and in the presence 30 of the vortices. In terms of the dimensionless parameters

$$
t=\frac{4 \pi}{g^{2}} T, \quad \tilde{\mu}=\mu a^{2}, \quad \tilde{\zeta}=\zeta a^{2}
$$

the lowest order renormalization group equations read

$$
\begin{aligned}
& \frac{d t}{d \lambda}=\pi^{2}\left(\tilde{\mu}^{2}-t^{2} \tilde{\zeta}^{2}\right) \\
& \frac{d \tilde{\mu}}{d \lambda}=\left(2-\frac{1}{t}\right) \tilde{\mu} \\
& \frac{d \tilde{\zeta}}{d \lambda}=(2-t) \tilde{\zeta}
\end{aligned}
$$


The fixed point structure of these equations is simple.

1. The point $T_{0}$

$$
t=0, \quad \tilde{\mu}=0, \quad \tilde{\zeta}=\infty
$$

is clearly the zero temperature fixed point. Here the long distance physics is dominated by the monopole induced mass term.

2. The point $T_{\infty}$

$$
t=\infty, \quad \tilde{\mu}=\infty, \quad \tilde{\zeta}=0
$$

is the high temperature fixed point. Here the infrared properties are determined by the charged plasma effects.

3. The point $T_{\mathrm{GG}}$

$$
t=1, \quad \tilde{\mu}=\tilde{\zeta}, \quad \tilde{\mu}=\infty
$$

is the infrared unstable fixed point. This is precisely the critical point that corresponds to the deconfining phase transition. At this point both the monopole and the charge plasma induced terms are equally important.

Note that the fixed line $t>2, \quad \tilde{\zeta}=0$ of massless theories which corresponds to the "confined monopole plasma" is not present, since for $t>2$ the charged plasma induced mass term is strongly relevant. The same is true for the would be fixed line $t<1 / 2, \tilde{\mu}=0$, which is present in the absence of the monopoles and describes the low temperature massless phase of noncompact electrodynamics.

It is instructive to see how the renormalization group equations formalize our qualitative arguments of the previous section. In particular, they make clear the role of the point $t=1 / 2$ which, in the absence of monopoles, would be the point where the charged induced term becomes relevant. If one starts the evolution from the initial condition $t=1 / 2, \tilde{\mu} \gg \tilde{\zeta}$, the running temperature will increase, and $\tilde{\mu}$ will grow into the infrared, while $\tilde{\zeta}$ will grow for a while until the running temperature reaches two. From this point on, $\tilde{\zeta}$ will decrease and the system will approach the high temperature fixed point. This corresponds to the situation where at the would be critical point $t=1 / 2$, the density of charged plasma is so high that the mean distance between the particles is smaller than the width of the confining string.

On the other hand, if the initial condition is $\tilde{\mu}<\tilde{\zeta}$, the temperature starts decreasing, making the coupling $\tilde{\mu}$ immediately irrelevant. Thus, $\tilde{\mu}$ monotonically decreases to zero and does not significantly affect the flow of the other couplings, which steadily flow to the zero temperature fixed point. This holds in the situation where at $t=1 / 2$ the density of charged plasma is low, which is indeed true in our model. 
Interestingly enough, at $t=1$ the initial conditions in our model in the case of a very heavy Higgs are such that the renormalization group flow starts almost exactly in the region of attraction of the fixed point $T_{\mathrm{GG}}$. At this value of temperature the monopole and charge fugacities are equal. The only difference between $\tilde{\mu}$ and $\tilde{\zeta}$ is then in the prefactors, which are possibly different combinations of $M_{W}$ and $g^{2}$. If $\tilde{\mu}$ and $\tilde{\zeta}$ were exactly equal, the only thing that happens along the flow is that their values grow, but $t$ does not change. Due to the small difference their values are equal at a temperature which slightly differs from 1 ,

$$
\frac{M_{W}}{T}=\frac{4 \pi M_{W}}{g^{2}}+O(1), \quad T=\frac{g^{2}}{4 \pi}\left[1+O\left(\frac{g^{2}}{M_{W}}\right)\right] .
$$

Thus, the initial temperature for which the system is in the region of attraction of $T_{\mathrm{GG}}$ is slightly different. We conclude that the critical temperature of the Georgi-Glashow model is indeed given by $T_{\mathrm{GG}}=\frac{g^{2}}{4 \pi}$, up to corrections of order $g^{2} / M_{W}$.

\subsection{The universality class of the phase transition}

Since the deconfining phase transition is due to the restoration of $Z_{2}$ symmetry, we expect that it is in the two-dimensional Ising universality class. This can be shown exactly by studying the theory (146) at the fixed point $t=1$. The following discussion follows closely Ref. 31. [See also Chapter 21 in the book 32.

The theory described by Eq. (146) can be fermionized by using the standard bosonization/fermionization techniques. Since at $t=1$ both cosine terms have dimension 1 , the resulting fermionic theory is a theory of free massive fermions. In our notations, the Dirac fermionic field is defined as

$$
\psi_{R}=a^{-1 / 2} i \exp \left[I\left(\chi+\frac{\tilde{\chi}}{2}\right)\right], \quad \psi_{L}=a^{-1 / 2} \exp \left[-I\left(\chi-\frac{\tilde{\chi}}{2}\right)\right] .
$$

The kinetic term in Eq. (146) then becomes the kinetic term of the field $\psi$, while the cosine terms become

$$
\begin{aligned}
& a^{-1} \cos 2 \chi=i\left[\psi_{R}^{\dagger} \psi_{L}-\text { H.c. }\right], \\
& a^{-1} \cos \tilde{\chi}=i\left[\psi_{R}^{\dagger} \psi_{L}^{\dagger}-\text { H.c. }\right] .
\end{aligned}
$$

Thus, the mass term in the fermionized Lagrangian is diagonalized by introducing the real Majorana fermions

$$
\rho=\frac{\psi+\psi^{\dagger}}{\sqrt{2}}, \quad \sigma=\frac{\psi-\psi^{\dagger}}{i \sqrt{2}} .
$$


The mass of the fermion $\rho$ is $\mu a+\zeta a$, while the mass of $\sigma$ is $\mu a-\zeta a$. For $\mu=\zeta$ the model contains one massive and one massless fermion. At the fixed point $\tilde{\mu} \rightarrow \infty$ the massive fermion decouples. Thus, at the point $T_{\mathrm{GG}}$ the theory is that of one massless Majorana fermion. It is well know that this theory precisely describes the critical point of the 2D Ising model.

Thus, we indeed see that the phase transition is in the universality class of the 2D Ising model.

\section{Vortices and monopoles at high temperature}

It is interesting to see how the behavior of magnetic vortices and the monopoles is affected by the deconfining phase transition. We will do this with the help of the effective action written in terms of the zeroth component of the vector potential $A_{0}$. The relation between our analysis, which was performed for the phase of the vortex operator $\chi$ and this standard procedure is not difficult to see. The free energy of the charged $W^{ \pm}$bosons in the Lagrangian (146) is given by the term $\cos \tilde{\chi}$. On the other hand, this same free energy in the standard calculation is represented by the insertion of the Polyakov line with charge two. Thus, we identify the Polyakov line with the exponential of the dual field

$$
P=\exp \left(\frac{i}{2} \tilde{\chi}\right)
$$

and the dual field $\tilde{\chi}$ with the Abelian vector potential

$$
\tilde{\chi}=2 g \beta A_{0} .
$$

Up to the monopole induced term, the Lagrangian (146) is equivalent to the sine-Gordon theory of the field $\tilde{\chi}$ in Eq. (140). With the identification in Eq. (168), this is

$$
\frac{2}{T}\left(\partial_{i} A_{0}\right)^{2}+\mu \cos \left(\frac{2 g}{T} A_{0}\right) .
$$

The monopole induced term also can be written in terms of the vector potential. Its form in terms of the Polyakov line $P$ is precisely the same as the form of the charge plasma induced term in terms of the vortex field $V$. Recall that the origin of the plasma induced term $\cos \chi$ in Eq. (146) is the dimensionally reduced "Skyrme term" of Eq. (35). Thus, just like we derived the Sine-Gordon Lagrangian (146) starting from the effective Lagrangian for the vortex field, we can follow the same steps backwards, but this time expressing everything in terms of $P$. Due to this duality between $V$ and $P$ we conclude that the 
monopole induced term is the dimensionally reduced Skyrme term for the Polyakov line. The full effective Lagrangian for the vector potential is then

$$
\mathcal{L}=\frac{2}{T}\left(\partial_{i} A_{0}\right)^{2}+\mu \cos \left(\frac{2 g}{T} A_{0}\right)+\frac{a^{2}}{4 \pi^{2}} \ln (\tilde{\zeta})\left(\epsilon_{i j} \partial_{i} P \partial_{j} P^{*}\right)^{2} .
$$

The monopole in this Lagrangian is represented by a vortex of the field $\frac{g}{T} A_{0}$ with unit vorticity. The coefficient of the Skyrme term in Eq. (170) is such that the action of such a unit vortex is equal to the action of the "core" of the monopole.

The cosine term in this expression is the potential for $A_{0}$ induced by the nonvanishing density of the charged particles in the thermal ensemble. Naturally, it contains the Debye "electric" mass term for $A_{0}$ and also higher interactions. Note that, as opposed to strongly interacting theories, where similar effective Lagrangians have been derived only in the derivative expansion, the Lagrangian (170) is valid on all distance scales longer than $1 / T$. Thus, in principle, it can be used to calculate correlation functions in a large momentum range. However, if one is interested in the long distance behavior of the correlators, at temperatures above $T_{\mathrm{GG}}$, the higher derivative Skyrme term can be neglected. This is in accordance with our analysis of the previous section, which showed that the monopole induced term is irrelevant above $T_{\mathrm{GG}}$.

Consider now the correlation function of two vortex operators. Recall that in the confining phase the vortex operator has a nonvanishing expectation value since the magnetic $Z_{2}$ symmetry is spontaneously broken. In the deconfined phase we expect the correlation function of two vortex fields to decay exponentially at large distance.

Consistent with the magnetic symmetry breaking, at zero temperature we have

$$
\left\langle V(x) V^{*}(y)\right\rangle=\frac{g^{2}}{8 \pi^{2}} \exp \left[-\frac{1}{2}\langle\chi(x) \chi(y)\rangle\right]=\frac{g^{2}}{8 \pi^{2}} \exp \left[-\frac{16 \pi^{2}}{g^{2}} Y_{3}(x-y)\right],
$$

where $Y_{3}(x-y)$ is the $3 \mathrm{~d}$ Yukawa potential with the mass $M$. At temperatures below the phase transition $\left(T<T_{\mathrm{GG}}\right)$ but high enough so that the dimensional reduction is valid the infrared asymptotics (at distances $|x-y|>1 / T$ ) is instead

$$
\left\langle V(x) V^{*}(y)\right\rangle=\frac{g^{2}}{8 \pi^{2}} \exp \left[-\frac{16 \pi^{2} T}{g^{2}} Y_{2}(x-y)\right]
$$

with $Y_{2}(x-y)$ the two-dimensional Yukawa potential with the mass which includes the effects of integrating out the nonzero Matsubara modes, as calculated in Ref. 25. This expression follows from Eq. (146) neglecting the $\cos \tilde{\chi}$ term, which is indeed negligible in the infrared. 
At high temperatures $T>T_{\mathrm{GG}}$ it is convenient to use Eq. (170) with the omission of the third term. The calculation of the correlator of the vortex operators proceeds along the lines of Refs. 33, 11. The insertion of $V(x)$ and $V^{*}(y)$ creates the $Z_{2}$ domain wall stretching between the points $x$ and $y$. In terms of the sine-Gordon theory, Eq. (170) this domain wall is just the kink, and thus the domain wall tension is equal to the soliton mass $M_{s}$. The correlator is then

$$
\left\langle V(x) V^{*}(y)\right\rangle=\exp \left\{-M_{s}|x-y|\right\}
$$

with the soliton mass

$$
M_{s}=a^{-1} \frac{2 \Gamma(p / 2)}{\sqrt{\pi} \Gamma\left(\frac{p+1}{2}\right)}\left[\frac{\pi \Gamma\left(\frac{1}{p+1}\right)}{\Gamma\left(\frac{p}{p+1}\right)} 2 \tilde{\mu}\right]^{\frac{p+1}{2}},
$$

where

$$
p=\frac{g^{2}}{8 \pi T-g^{2}} .
$$

Thus, we find that the correlation function of the vortex operators decreases exponentially in the high temperature phase as it should in the phase with restored symmetry. Note that, were we to neglect the Debye mass term, we would have found that below $T_{\mathrm{BKT}}$ the correlator tends to a constant at infinity, while at $T>T_{\mathrm{BKT}}$ it decays at large distances, but only as a power, since the mass of the soliton vanishes.

What about the monopoles? The discussion in the beginning of this section would suggest that they are "confined" logarithmically. However, it is easy to see from the effective action Eq. (170) that the monopoles are bound by a (screened) linear potential. Consider a configuration with unit winding of the field $P$. Due to the potential term $P^{2}$, the minimal action configuration cannot be a rotationally symmetric hedgehog. Such a configuration would "cost" action proportional to the volume, since the field $P$ would be away from its vacuum value everywhere in space. The best one can do is to have a quasi one dimensional strip in which the winding is concentrated, while everywhere else in space $P$ would be equal to 1 (or -1 ). This configuration is schematically depicted in Fig. 4.

The width of the "dual confining string" must clearly be of order of $M_{D}^{-1}$, while the action per unit length, $s \propto \frac{T}{g^{2}} M_{D}$. Thus, the action of a single monopole diverges linearly with the size of the system. Obviously the monopole-anti-monopole pair separated by a distance $L$ have action $L s$. When the distance is large enough, another pair can be produced from the thermal ensemble, screening the linear potential. The critical distance at which this 


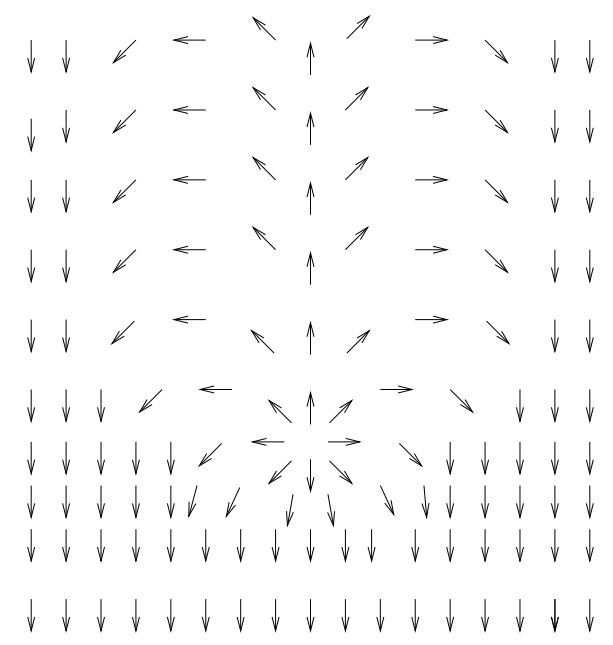

Figure 4: The string-like configuration of the Polyakov line $P$ that accompanies the monopole-instanton in the high temperature phase.

happens is determined by

$$
L_{c} s=2 \log \zeta=\frac{8 \pi M_{W}}{g^{2}}
$$

or

$$
L_{c} \propto \frac{M_{W}}{T} M_{D}^{-1}
$$

Thus, as long as the temperature is much lower than $M_{W}$, the length of the "dual string" is much greater than its thickness. The "potential" between the monopoles is therefore linear, but screened at large distances, much the same as the confining potential in gauge theories with heavy fundamental charges. Thus, the behavior of the monopoles is in many senses "dual" to that of electric chargese.

Even though the monopoles are "confined", their effects do not disappear in the hot phase. They, for example, are the main contributors to the correlation functions of the vortex operators, of the type $V(x) V(y)$. This correlator would be vanishing if the magnetic symmetry were $\mathrm{U}(1)$ rather than $Z_{2}$. It was shown, however, in Ref. 34, that this correlation function does not vanish

${ }^{e}$ Of course one always has to keep in mind that physically monopoles and charges are very different objects in this model: the charges are particles, while the monopoles are instantons. 
due to the monopole contributions. In fact, the "diagonal" correlation functions are those of the parity even and parity odd operators $V^{+}=V+V^{*}$ and $V^{-}=V-V^{*}$

$$
\begin{aligned}
& \left\langle V^{+}(x) V^{+}(y)\right\rangle=\left(a M_{D}\right)^{\frac{\pi T}{g^{2}}} \exp \left\{-|x-y| \tilde{\sigma}_{-}\right\} \\
& \left\langle V^{-}(x) V^{-}(y)\right\rangle=\left(a M_{D}\right)^{\frac{\pi T}{g^{2}}} \exp \left\{-|x-y| \tilde{\sigma}_{+}\right\}
\end{aligned}
$$

with

$$
\tilde{\sigma}_{ \pm}=M_{s} \pm \zeta\left(a M_{D}\right)^{\frac{2 \pi T}{g^{2}}-1} \frac{1}{\pi a} .
$$

Thus, the correlation lengths in the scalar and pseudoscalar channels are not equal due to the monopole effects. At high temperatures this difference becomes small, since $\zeta \ll a M_{D} \ll 1$, but it nevertheless remains strictly finite at any finite temperature.

\section{Hot confining strings: Deconfinement versus Hagedorn transi- tions}

In this section we discuss the deconfining phase transition from the perspective of the confining string.

What happens to strings at very high temperatures is one of the big questions of string theory. In the early days of dual resonance models and hadronic bootstrap, the exponential growth in the one-particle density of states

$$
\rho(m) \sim m^{a} e^{b m}
$$

led to the famous Hagedorn transition 35 This type of spectrum first arose in the context of statistical bootstrap models $3,36,37$ and, for hadrons, such behavior indicates that they are composed of more fundamental constituents 38 In fundamental string theories one finds the same kind of spectrum (see, for example, books 39), and a search for hints to the existence of "string constituents is of great interest. What lies beyond the Hagedorn temperature? Is this temperature limiting or is there a high-temperature phase which reveals the fundamental degrees of freedom? And is it true that for all types of string theories there is the same universal physics or is it that different classes may have totally different high-temperature behavior? A lot of effort has been invested into study of the Hagedorn transition in critical (super)strings. There is an enormous volume of literature on this subject, some references (but by no means all) can be found, for example, in Ref. 40. For weakly coupled critical (super)strings the Hagedorn transition can be described as a 
Berezinsky-Kosterlitz-Thouless 28 transition on a world sheet $41,42,43,44$ It is due to the "condensation" of the world sheet vortices. It has been also suggested that the transition in some cases may actually be first order. Above the Hagedorn temperature, the vortices populate the world sheet and we have a new phase. From the target space point of view we are talking about tachyonic instabilities for non zero winding modes in the imaginary time direction (thermal winding modes).

In the more general case of interacting strings, one does not know with certainty what the fundamental degrees of freedom are and thus what their role is at high temperature. Recently it was suggested, in the framework of the Matrix Model description of the Hagedorn transition, that the fundamental string decays into D0 Branes 45 Thus, it could be that D0 branes are the fundamental degrees of freedom in the hot phase.

In short, there are many interesting open string theory questions pertaining to the hot string. Since our simple $2+1$ dimensional model is well understood, it is worth seeing what it has to say on these issues.

\subsection{The monopole binding as the Hagedorn transition}

As we saw in the previous sections, the dynamics of the deconfining phase transition is quite nontrivial, involving interplay between the charged degrees of freedom and the monopoles. To disentangle these effects in string language, we start our discussion by completely disregarding the charged particles. From the string point of view, this means that we neglect possible contributions of the heavy D0 branes, and are thus entirely within the theory of closed strings. Naively one expects in such a theory the existence of a Hagedorn temperature, beyond which the string cannot exist. In an almost free string this temperature is of order of the string scale. One can visualize this phenomenon in simple terms. Consider a closed string of a given fixed length $L$. Let us calculate the free energy of such a string. The energy of the string is

$$
E=\sigma L
$$

The number of states for a closed string of length $L$ scales exponentially with $L$

$$
N(L)=\exp \{\alpha L\}
$$

The dimensional constant $\alpha$ is determined by the physical thickness of the string. Imagine that the string can take only positions allowed on a lattice with the lattice spacing $a$. Then clearly the number of possible states is $z^{a} L$, where $z$ is a number of order unity, equal to the number of nearest neighbors 
on the lattice.f In this simple situation $\alpha=a \ln z$. The only natural lattice spacing for such a discretization is the thickness of the string. For an almost free string the thickness is naturally the same as the scale associated with the string tension. Thus, the entropy is

$$
S=x \sqrt{\sigma} L
$$

with $x$ a number of order unity. The free energy is then

$$
F[L]=\sigma L-x T \sqrt{\sigma} L
$$

At the temperature

$$
T_{H}=\frac{1}{x} \sqrt{\sigma}
$$

the free energy becomes negative, which means that strings of arbitrary length appear in the thermal ensemble in a completely unsuppressed way. The thermal vacuum becomes a "soup" of arbitrarily long strings. Thus, effectively, the "temperature dependent" string tension vanishes and it is not possible to talk about strings any more in the hot phase. For more details about this "random walk" description of hot strings, see Ref. 46 and references therein.

The situation is very similar to the BKT phase transition, where the free energy of a vortex becomes negative at the critical temperature, and the vortices populate the vacuum in the hot phase.

In the string partition function language this is just a restatement of the well known fact that the partition function diverges in a sector with the topology of a torus which winds around the compact Euclidean time direction. Fixing the unit winding in the Euclidean time physically corresponds to the calculation of the free energy in the sector with one closed string. The integration over all possible lengths of the string is the cause of the divergence of the partition function at high temperature.

The same physical effect exists for the confining Georgi- Glashow string. There is one difference, which however turns out to be crucial for the nature of the phase transition. This is that the thickness of the Georgi-Glashow string is not given by the string tension. Rather it is equal to the inverse photon mass, and thus

$$
\alpha \propto M_{\gamma} \propto \frac{\sigma}{g^{2}}
$$

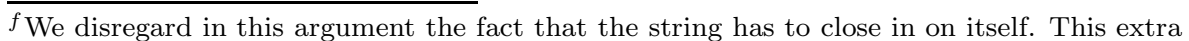
condition would lead to a pre-factor with power dependence on $L$. Such a pre-factor is not essential for our argument, and we therefore do not worry about it. 
We thus have for the confining Georgi-Glashow string

$$
T_{H}=\frac{\sigma}{\alpha} \propto g^{2} .
$$

This is indeed the correct magnitude of the critical temperature as discussed in previous sections.

The noteworthy feature of this formula is that the Hagedorn temperature of the confining string is much higher than the string scale. This is easy to understand because the entropy of the thick string is much smaller than that of the free string due to the fact that high momentum modes of the confining string do not contribute to the entropy at all, as discussed earlier. Thus, one needs to heat the string to a much higher temperature for entropy effects to become important.

Since we have completely neglected the possibility of the appearance of D0 branes (charged particles), the transition we have been discussing is the string representation of the monopole binding transition in the Georgi-Glashow model. At the point where the monopoles bind, the photon of the GeorgiGlashow model becomes massless and thus the string tension disappears and the thickness of the string diverges. In the Hagedorn picture this is just the dual statement that the ensemble is dominated by infinitely long strings. The BKT nature of both transitions underlines this point.

\subsection{Vortices on the world sheet - open strings and charged particles}

Sometimes the Hagedorn transition is discussed in terms of the vortices on the world sheet. What is the physical nature of these objects?

Consider a string world sheet with the topology of a sphere with a vortexanti-vortex pair, Fig. 5. When going around the vortex location on the world sheet, the compact coordinate $x_{0}$ varies from 0 to $\beta$. Since this is true for any contour of arbitrarily small radius, which encircles the vortex, this means that physically the location of the vortex in fact corresponds in the target space not to a point but rather to a line which winds around the compact direction. The vortex-anti-vortex pair on the world sheet thus represents an open string which winds around the compact direction. Figure 5 illustrates how the open string world sheet, equivalent to a cylinder is transformed by a conformal transformation into a sphere with two singular points - the vortexanti-vortex pair.

If the string theory in question does have an open string sector, then the configurations with arbitrary number of vortex-anti-vortex pairs contribute to the finite temperature partition function. The Hagedorn phase transition can then be discussed in this sector rather than in the closed string sector. Not 
A
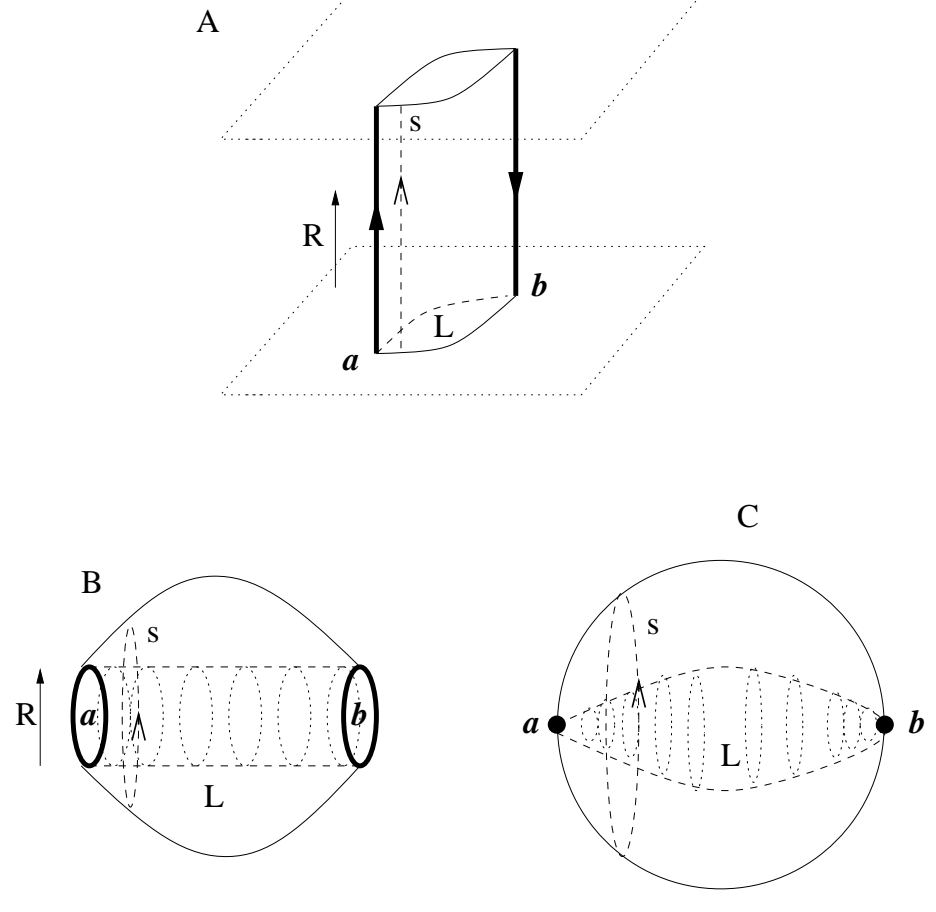

Figure 5: Representation of a vortex and an anti-vortex pair with a string stretched between them and the corresponding string world sheets. 
surprisingly, the discussion is exactly the same as in the previous subsection. The fact that we now have an open rather than closed string does not change the entropy versus energy argument. One finds that at $T<T_{H}$ the vortices on the world sheet are bound in pairs. The corresponding target space picture is that the "ends" of the open strings are bound by a linear potential and therefore long open strings do not contribute to the thermal ensemble. In exactly the same way, long closed strings are also absent from the ensemble. At $T>T_{H}$ the vortices unbind and appear in the ensemble as a Coulomb gas. Thus, a typical configuration contains lots of open strings (as well as lots of arbitrarily long closed strings) since the energy of such strings is overwhelmed by the entropy.

Note, however, that the existence of the transition in this context is entirely independent of the presence or absence of vortices. As we saw above, the transition can be understood purely on the level of the closed string. It is driven by the string fluctuations. Thus, even if the theory does not have an open string sector, the transition is still there. For example there are no open strings in the Georgi-Glashow model. Nevertheless, if we neglect the effects of $W^{ \pm}$the Hagedorn transition is still there and it coincides with the "monopole binding" transition.

The actual phase transition in the Georgi-Glashow model is however not driven by monopole binding. Nevertheless, in the string language it is also due to proliferation of vortices on the world sheet. These are, however, vortices of a somewhat different type. The same conformal transformation that turned the open string boundary into a point can be used to turn the world line of a heavy particle into a point - let's call it 0 brane. As discussed above, this is a (fundamentally) charged particle which couples to the Georgi-Glashow confining string. Thus, a string world sheet representation of a pair of particles with opposite charge is also a vortex-anti-vortex pair on a sphere.

There are some important differences between these vortices and the ones that represent the ends of the open string. First, for an open string the noncompact "spatial" coordinates satisfy Neumann boundary conditions. Thus, even as $x^{0}$ winds, the other coordinates $x^{i}$ can take arbitrary values close to the vortex. One can see this from the action

$$
S=\sigma \int d^{2} \xi \partial_{a} x^{0} \partial_{a} x^{0}+\sigma \int d^{2} \xi \partial_{a} x^{i} \partial_{a} x^{i}
$$

where the dynamics of $x^{i}$ is absolutely unrelated to the dynamics of $x^{0}$. Thus, even if a vortex in $x^{0}$ sector can be considered as a boundary in a target space, there are free boundary conditions for the components $x^{i}$. In other words, we do not have any boundary action induced by vortices in a theory of closed 
strings.

For a very heavy 0 brane the boundary conditions are of the Dirichlet type. Thus, $x_{i}$ are constant close enough to the vortex location. Another difference is that, since the 0 brane is an independent degree of freedom, in principle its mass is a free parameter. Thus, the fugacity of the 0 brane vortex is an independent parameter, and physics may in principle depend on it. Of course, in the case when we have a finite mass for 0 branes there is a nontrivial boundary action describing the massive particle. In that case one has pen-conformal boundary conditions compatible with a finite mass of a 0 brane 4

The Georgi-Glashow confining string has neither open string sectors nor 0 branes which are the sources of a single string. The dynamical objects which couple to the string are the charged $W^{ \pm}$particles, which have an adjoint charge and therefore are sources of a pair of strings.

Each dynamical 0 brane in the Georgi Glashow model has two strings emanating from it. Thus, a pair of branes propagating in compact imaginary time is conformally equivalent to a pair of "double vortices" as in Fig. 6.

The singular points are still vortices as before, since going around such a point one travels once around the compact time direction. But now two string world sheets are permanently glued together at the location of a vortex. The fact that two world sheets are glued at the location of the vortex is the manifestation of the $Z_{2}$ magnetic symmetry of the Georgi- Glashow model. The region of space between the two world sheets is separated from the region of space on the outside, reflecting the fact that it constitutes a domain of a different vacuum.

At low temperatures such configurations in the thermal ensemble are rare. But at the critical temperature their density becomes so large that the ensemble is dominated by multi-vortex configurations. In the string picture these are configurations with multiple points of "gluing". Importantly, the 0 branes are not just pairwise connected by two strings, but, rather, form a network where all of them are connected to each other. Clearly in terms of entropy such configurations are much more favorable, and there is also no loss of energy when the distance between the 0 branes is of the order of the string thickness.

This string-based description of the transition has to be taken with a grain of salt. The relevant physics takes place on short distance scales - of the order of the thickness of the string. On these scales, as we explained above, the string modes are practically absent. Thus, even though we have showed the string segments on Fig. 6, these segments are so short that there is no string tension associated with them. Thus, the mechanism of the transition is essentially field-theoretical rather than string-theoretical.

To understand this point better consider in a little more detail the thermal 

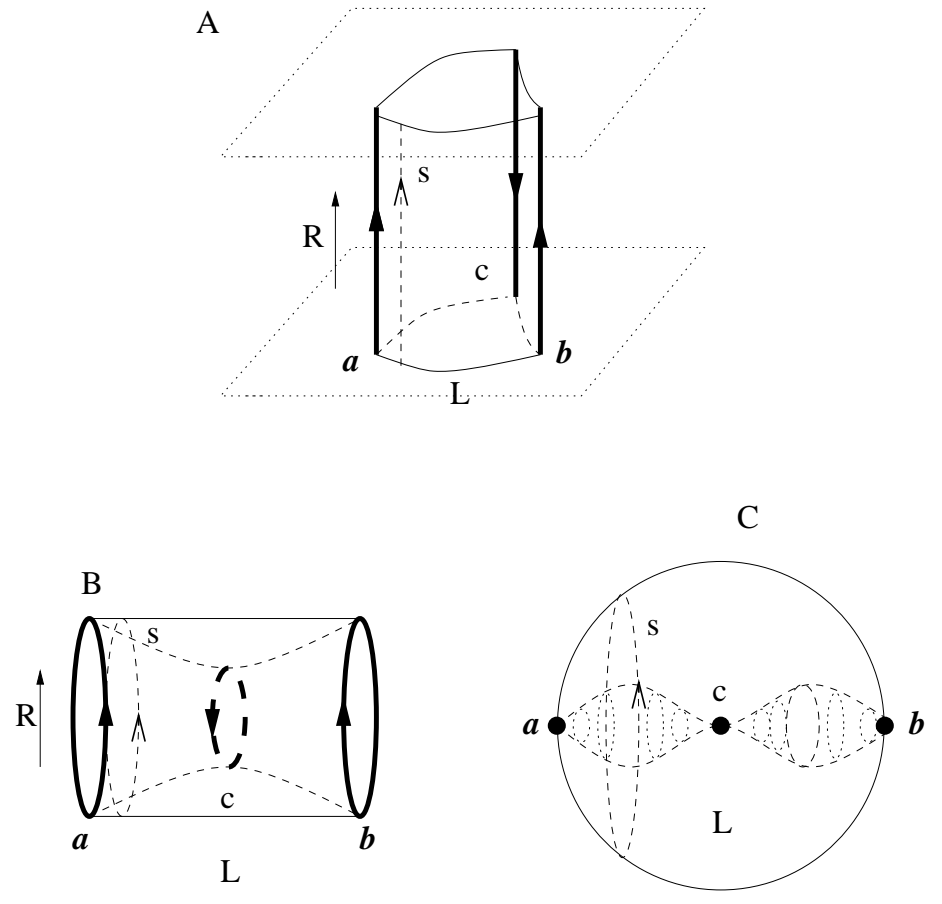

Figure 6: See the text 
ensemble of $W^{ \pm}$. The crucial point is that at distance scales $d \ll M_{\gamma}^{-1}$ the interaction between them is Coulomb rather than linear. The gas of charges with Coulomb interaction has itself a transition into a plasma phase. This transition has nothing to do with the long range linear interaction and occurs at the temperature $T_{N C}$ which is four times smaller than the Hagedorn temperature 26 At this temperature the $W^{ \pm}$become "free" in the sense that they cease to care about the Coulomb part of the potential. The crucial question however is: how large is the density of $W^{ \pm}$at this point? If the density at $T_{N C}$ were large and the average distance between $W$ 's were smaller than $M_{\gamma}^{-1}$, the transition would actually occur at $T_{N C}$, since the long range linear part of the potential would be entirely irrelevant. As it happens, in the Georgi-Glashow model this is not the case, and the density of $W$ is small. Thus, at $T_{N C}$ there is a certain rearrangement of the thermal ensemble on short distance scales, but at long distances nothing happens - the string still confines. However, at $T_{C}=T_{H} / 2$ the density of $W$ reaches the critical value and the transition occurs. Note that at this temperature the large length fluctuations of the string are still suppressed - we are far below the Hagedorn temperature. The string is destroyed not due to "stringy" physics of the Hagedorn transition, but due to the short distance field-theoretical effects, viz. the fact that the fugacity of $W$ is relatively large and that the interaction at short distances is Coulomb and not linear.

\section{The $\mathbf{S U}(N)$ model}

So far in this review we have discussed the SU(2) gauge theory. Most of the discussion is easily generalized to the weakly interacting theory with $\mathrm{SU}(N)$ gauge group,

$$
\mathcal{L}=-\frac{1}{2} \operatorname{tr} F_{\mu \nu} F^{\mu \nu}+\operatorname{tr} D_{\mu} \Phi D^{\mu} \Phi-V(\Phi),
$$

where

$$
\begin{aligned}
A_{\mu} & =A_{\mu}^{a} T^{a}, & F_{\mu \nu}=\partial_{\mu} A_{\nu}-\partial_{\nu} A_{\mu}+g\left[A_{\mu}, A_{\nu}\right], \\
\Phi & =\Phi^{a} T^{a}, & D_{\mu} \Phi=\partial_{\mu} \Phi+g\left[A_{\mu}, \Phi\right] .
\end{aligned}
$$

Here $T^{a}$ are traceless Hermitian generators of the $\mathrm{SU}(N)$ algebra normalized such that $\operatorname{tr}\left(T^{a} T^{b}\right)=\frac{1}{2} \delta^{a b}$.

Depending on the form of the Higgs potential, there can be different patterns of gauge symmetry breaking. Since most of the details of the potential are unimportant for our purposes, we will not specify it except for restricting 
it to the region of the parameter space where classically the gauge symmetry is broken to the maximal torus

$$
\mathrm{SU}(N) \rightarrow \mathrm{U}(1)^{N-1}
$$

We also restrict ourselves to the weakly coupled regime, which means that the ratios $M_{W} / g^{2}$ are large for all $N^{2}-N$ massive $W$ bosons.

To characterize the perturbative spectrum of the theory it is convenient to use the Cartan-Weyl basis $\left(H^{i}, E^{\vec{\alpha}}\right)$, where $H^{i}$ generate the Cartan subalgebra which is of dimension of the rank $r=N-1$ of $\mathrm{SU}(N)$,

$$
\left[H^{i}, H^{j}\right]=0, \quad i, j \in[1,2, . . N-1],
$$

and $E^{\vec{\alpha}}$ are the $N(N-1)$ ladder operators which satisfy

$$
\begin{aligned}
{\left[H^{i}, E^{\vec{\alpha}}\right] } & =\alpha^{i} E^{\vec{\alpha}}, \\
{\left[E^{\vec{\alpha}}, E^{\vec{\beta}}\right] } & =N_{\vec{\alpha}, \vec{\beta}} E^{\vec{\alpha}+\vec{\beta}} \quad \text { if } \vec{\alpha}+\vec{\beta} \text { is a root } \\
& =2 \vec{\alpha} \cdot \vec{H} \quad \text { if } \quad \vec{\alpha}=-\vec{\beta} .
\end{aligned}
$$

The $(N-1)$-dimensional root vectors $\vec{\alpha}=\left(\alpha^{1}, \alpha^{2}, \ldots \alpha^{N-1}\right)$ form the dual Cartan subalgebra. There are obviously $N(N-1)$ such vectors corresponding to $\operatorname{dim}(\mathrm{SU}(N))-\operatorname{rank}(\mathrm{SU}(N))$ but only $N-1$ of them are linearly independent. The non-vanishing inner products in the Cartan-Weyl basis are

$$
\operatorname{tr}\left(H^{i}, H^{j}\right)=\frac{1}{2} \delta^{i j}, \quad \operatorname{tr}\left(E^{\vec{\alpha}}, E^{\vec{\beta}}\right)=\frac{1}{2} \delta^{\vec{\alpha},-\vec{\beta}} .
$$

At the classical level $N-1$ gauge group generators are unbroken, which we choose to correspond to $\left\{H^{i}\right\}$. Therefore, classically there are $N-1$ massless photons and $N(N-1)$ charged massive $W$ bosons.

Our Weyl basis is chosen in such a way that the Higgs VEV is diagonal. Since the matrix $\Phi$ is traceless, there are $N-1$ independent eigenvalues. In terms of the $N-1$ dimensional vector $\vec{h}=\left(h_{1}, h_{2}, h_{3}, . . h_{N-1}\right)$ we have?

$$
\langle\Phi\rangle=\vec{h} \cdot \vec{H}, \quad A_{\mu}=\vec{A}_{\mu} \cdot \vec{H}+\sum_{\vec{\alpha}} A_{\mu}^{\vec{\alpha}} E^{\vec{\alpha}}
$$

${ }^{g}$ For concreteness we order these numbers $h_{1}>h_{2}>\ldots h_{N-2}>h_{N-1}$, which also breaks the discrete Weyl group. 
For concreteness let us choose the following basis for the Cartan subalgebra:

$$
\begin{gathered}
H_{1}=\frac{1}{2} \operatorname{diag}(1,-1,0, \ldots 0), \quad H_{2}=\frac{1}{2 \sqrt{3}} \operatorname{diag}(1,1,-2,0 \ldots 0) \\
\ldots \quad H_{N-1}=\frac{1}{\sqrt{2 N(N-1)}} \operatorname{diag}(1,1,1, \ldots 1,-(N-1)) .
\end{gathered}
$$

As long as $\vec{h} \cdot \vec{\alpha} \neq 0$ for all roots, the gauge symmetry is maximally broken. The masses of the $W$ bosons can be read off from the second term in the Lagrangian

$$
\begin{aligned}
& g^{2} \operatorname{tr}\left[A_{\mu}, \Phi\right]^{2}=\frac{g^{2}}{2} \sum_{\vec{\alpha}, i, j} A_{\mu}^{\vec{\alpha}} A_{\mu}^{-\vec{\alpha}} h_{i} h_{j} \alpha^{i} \alpha^{j} \\
& \Longrightarrow M_{\vec{\alpha}}=g|\vec{h} \cdot \vec{\alpha}| .
\end{aligned}
$$

The $W$ bosons corresponding to the $N-1$ simple roots $\vec{\beta}_{i}, \quad i=1, \ldots, N-1$ (an arbitrarily chosen set of linearly independent roots) can be thought of as fundamental, in the sense that the quantum numbers and the masses of all other $W$ bosons are obtained as linear combinations of those of the fundamental $W$ bosons. These charges and masses are

$$
\vec{Q}_{\vec{\beta}}=g \vec{\beta}, \quad M_{\vec{\beta}}=g \vec{h} \cdot \vec{\beta} .
$$

As an example, consider the case of $\mathrm{SU}(3)$ broken down to $\mathrm{U}(1) \times U(1)$. There are 6 massive $W$ bosons. The simple roots can be taken as

$$
\vec{\beta}_{1}=\left(\frac{1}{2}, \frac{\sqrt{3}}{2}\right), \quad \vec{\beta}_{2}=\left(-\frac{1}{2}, \frac{\sqrt{3}}{2}\right) .
$$

The remaining non-simple positive root is

$$
\vec{\alpha}_{3}=\vec{\beta}_{1}-\vec{\beta}_{2}=(1,0) .
$$

The other three roots are $-\vec{\beta}_{i},-\vec{\alpha}_{3}$. The masses of corresponding $W$ bosons are

$$
M_{W_{1}}=\frac{g}{2}\left(h_{1}+\sqrt{3} h_{2}\right), \quad M_{W_{2}}=\frac{g}{2}\left(h_{1}-\sqrt{3} h_{2}\right), \quad M_{W_{2}}=g h_{1}
$$

for $h_{1}>\sqrt{3} h_{2}$. 


\subsection{The monopole-instantons and the Polyakov effective Lagrangian}

Non-perturbatively, the most important contributions in the theory are due to the monopole-instantons. Those are classical, stable, finite action solutions of the Euclidean equations of motion arising due to the nontrivial nature of the second homotopy group of the vacuum manifold $\left(\Pi_{2}\left(\mathrm{SU}(N) / \mathrm{U}(1)^{N-1}\right)=Z^{N}\right)$. The magnetic field of such a monopole is long range.

$$
B_{\mu}=\frac{x^{\mu}}{4 \pi r^{3}} \vec{g} \cdot \vec{H}
$$

The $N-1$ dimensional vectors $\vec{g}$ are determined by the non-Abelian generalization of the Dirac quantization condition 4 . 40

$$
e^{i g \vec{g} \cdot \vec{H}}=I .
$$

Solutions of this quantization condition take the form

$$
\vec{g}=\frac{4 \pi}{g} \sum_{i=1}^{N-1} n_{i} \vec{\beta}_{i}^{*}
$$

where $\vec{\beta}^{*}$ are the dual roots defined by $\vec{\beta}^{*}=\vec{\beta} /|\vec{\beta}|^{2}$. We will be working with roots normalized to unity, and thus $\vec{\beta}^{*}=\vec{\beta}$. The integers $n_{i}$ are elements of the group $\Pi_{2}$ (see Ref. 50). The monopoles which have the smallest action correspond to roots taken once. The action of these monopoles in the BPS limit is

$$
M_{\vec{\alpha}}=\frac{4 \pi}{g} \vec{h} \cdot \vec{\alpha}=\frac{4 \pi M_{W_{\vec{\alpha}}}}{g^{2}} .
$$

Just like with $W$ bosons we can think of monopoles corresponding to simple roots as fundamental ones with magnetic charges and action

$$
\overrightarrow{g_{i}}=\frac{4 \pi}{g} \vec{\beta}_{i} \quad M_{i}=\frac{4 \pi}{g} \vec{h} \cdot \vec{\beta}_{i} .
$$

For example, in the case of $\mathrm{SU}(3)$ (see Eqs. 202,204)) the monopole action spectrum (in the BPS limit) is

$$
M_{1}=\frac{2 \pi}{g}\left(h_{1}+\sqrt{3} h_{2}\right), \quad M_{2}=\frac{2 \pi}{g}\left(h_{1}-\sqrt{3} h_{2}\right), \quad M_{3}=\frac{4 \pi}{g} h_{1} .
$$

The effect of these monopoles is to impart finite mass to all the perturbatively massless "photons." The derivation of the effective Lasrangian follows exactly the same lines as the original derivation of Polyakov for the SU(2) 
theory. The resulting low-energy effective theory is written in terms of the $N-1$ component field, $\vec{\eta}$, with the following Lagrangian:51.52

$$
\mathcal{L}_{\text {eff }}=\frac{g^{2}}{32 \pi^{2}}\left(\partial_{\mu} \vec{\eta}\right)^{2}+\sum_{\alpha} \frac{M_{\alpha}^{2} g^{2}}{16 \pi^{2}} \exp (i \vec{\alpha} \cdot \vec{\eta})
$$

The sum is over all $N(N-1)$ non-vanishing roots. The potential induced by the monopoles is proportional to the monopole fugacity

$$
M_{\alpha}^{2}=\frac{16 \pi^{2} \xi_{\alpha}}{g^{2}}, \quad \xi_{\alpha}=\text { const. } \frac{M_{W_{\alpha}}^{7 / 2}}{g} \exp \left[-\frac{4 \pi M_{W_{\alpha}}}{g^{2}} \epsilon\left(\frac{M_{H}}{M_{W}}\right)\right] .
$$

At weak coupling the photons are much lighter than the $W$ bosons and thus are the only relevant degrees of freedom in the low-energy sector.

\subsection{The magnetic $Z_{N}$ symmetry}

The global symmetry structure is very important for the understanding of the deconfining transition. The relevant symmetry in the present model is the magnetic $Z_{N}$ symmetry. We now wish to explain how this symmetry is implemented in the effective low-energy Lagrangian - generalizing the result obtained for $Z_{2}$ in the previous section.

The order parameter of the magnetic symmetry is the set of magnetic vortex operators $V_{i}, i=1, \ldots, N-1$. These operators were constructed explicitly in Ref. 10. These operators carry the magnetic fluxes of the $N-1 \mathrm{U}(1)$ Abelian magnetic fields. The defining commutation relation for $V_{i}$ is

$$
\left[V_{i}(x), \vec{B}(y)\right]=-\frac{4 \pi}{g} \vec{w}_{i} V_{i}(x) \delta^{2}(x-y) .
$$

Here $\vec{B}$ is the $N-1$ dimensional vector of magnetic fields, whose $j$-th component is the projection of the non-Abelian field strength onto the direction of the Cartan subalgebra generator $H_{j}$, and $\vec{w}_{j}$ are $N-1$ weight vectors of $\mathrm{SU}(N)$. The choice of the $N-1$ out of $N$ weight vectors is arbitrary. A change in this choice will lead to the redefinition of the vortex operators such that the new operators will be products of the old ones and their conjugates. It is always possible to choose these weights so that together with the "fundamental" roots $\beta_{i}$ they satisfy the relation

$$
\vec{w}_{i} \vec{\beta}_{j}=\frac{1}{2} \delta_{i j}
$$

${ }^{h}$ These magnetic fields can be constructed in an explicitly gauge invariant way from the non-Abelian field strengths and the Higgs field, see Ref. 10. 
The flux eigenvalues in Eq. (213) are dictated by the requirement of the locality of the vortex operators and are analogous to the Dirac quantization condition. The explicit form of the vortex operators in terms of the field $\eta$ in Eq. (211) is

$$
V_{i}(\vec{x})=\frac{g}{\sqrt{8} \pi} e^{i \chi_{i}}
$$

with

$$
\chi_{i}=\vec{w}_{i} \cdot \vec{\eta} \Longrightarrow \vec{\eta}=2 \sum_{i} \vec{\beta}_{i} \chi_{i}
$$

The effective Lagrangian can be written as a nonlinear $\sigma$-model in terms of $V_{i}$ as

$$
\begin{aligned}
\mathcal{L}_{\text {eff }}= & \frac{N-1}{2} \sum_{i, j} A_{i j} \frac{1}{V_{k}^{*} V_{k}}\left(V_{i}^{*} \partial_{\mu} V_{i}\right)\left(V_{j} \partial_{\mu} V_{j}^{*}\right)+ \\
& \lambda\left(\sum_{i}\left(V_{i} V_{i}^{*}-\frac{g^{2}}{8 \pi^{2}}\right)^{2}+\sum_{\alpha} k_{\alpha} \prod_{i} V_{i}^{2 i \vec{\alpha} \cdot \vec{\beta}_{i}}\right.
\end{aligned}
$$

with $\lambda \rightarrow \infty$

The matrix $A_{i j}=2 \vec{\beta}_{i}^{*} \cdot \vec{\beta}_{j}$ depends on the choice of the fundamental roots. With the conventional choice of positive roots, where $\vec{\beta}_{i} \vec{\beta}_{j}=-1 / 2, \quad i \neq j$, it is the Cartan matrix of the Lie algebra. All its diagonal elements are equal to 2 , while all its off diagonal elements equal -1 . We will find it however more convenient in the following to use a different set of fundamental roots, for which $\vec{\beta}_{i} \vec{\beta}_{j}=1 / 2, \quad i \neq j$. Such a choice is always possible for any $\operatorname{SU}(N)$. For this choice of roots the off-diagonal matrix elements of $A_{i j}$ are all equal to 1.

For SU(3) we have

$$
\mathbf{A}=\left(\begin{array}{ll}
2 & 1 \\
1 & 2
\end{array}\right)
$$

and the effective Lagrangian

$$
\begin{aligned}
\mathcal{L}_{\text {eff }}=\quad & \partial_{\mu} V_{1} \partial_{\mu} V_{1}^{*}+\frac{8 \pi^{2}}{g^{2}} V_{1}^{*} \partial_{\mu} V_{1} V_{2} \partial_{\mu} V_{2}^{*}+\partial_{\mu} V_{2} \partial_{\mu} V_{2}^{*} \\
& +\xi_{1}\left(V_{1} V_{2}^{*}+\text { c.c }\right)+\xi_{2}\left(V_{1}^{2} V_{2}+\text { c.c }\right)+\xi_{3}\left(V_{1} V_{2}^{2}+\text { c.c }\right) .
\end{aligned}
$$

The magnetic $Z_{N}$ symmetry has an obvious and simple representation in this effective Lagrangian as

$$
V_{i} \rightarrow \exp \{2 \pi i n / N\} V_{I}
$$


As long as only small fluctuations of the phase fields $\chi_{i}$ are important, the Lagrangian (219) is equivalent to Eq. (211). Thus, at low temperature the descriptions based on these Lagrangians are equivalent. The difference appears just as for $\mathrm{SU}(2)$ only when the phase nature of $\chi_{i}$ plays a role. Indeed, since $\chi_{i}$ are treated in Eq. (219) as phases, dynamically one allows configurations in which these phases have nontrivial winding. On the other hand, in Eq. (211) such configurations cost an infinite amount of energy. As discussed in detail in Refs. 10 and 26 the winding configurations correspond to the heavy $W$ bosons. In fact, the explicit relation between the vorticity of the fields $V_{i}$ and the electric charges is given by 10

$$
\frac{1}{g} \vec{w}_{i} \vec{Q}=\frac{1}{4 \pi} \oint_{C \rightarrow \infty} d l_{\mu} \partial_{\mu} \chi_{\mu}^{i} .
$$

Thus, the difference between the two Lagrangians is important whenever the physics of the $W$ bosons plays an important role. We have seen in the case of the $\mathrm{SU}(2)$ theory that $W$ 's are indeed important near the phase transition temperature. The same turns out to be true for arbitrary $\mathrm{SU}(N)$. We this have to be careful to treat the $W$ bosons properly in the transition region 53

\subsection{The deconfining transition}

Close to the transition temperature we can safely use the dimensionally reduced Lagrangian. The zero Matsubara frequency sector is described by the twodimensional Lagrangian

$$
\mathcal{L}_{\text {eff }}=\frac{g^{2}}{32 \pi^{2} T}\left(\partial_{\mu} \vec{\eta}\right)^{2}+\sum_{\alpha} \frac{M_{\alpha}^{2} g^{2}}{16 \pi^{2} T} \exp (i \vec{\alpha} \cdot \vec{\eta}) .
$$

However, as we noted before, our description should include $W$ bosons, and so the fields $\eta$ should be treated here as phases with periodicity appropriate to Eq. (216). In fact, the Lagrangian also has to be augmented by a four derivative "Skyrme" term, which fixes the energy of the winding states to be equal to the masses of $W$ bosons 26 Things are however simplified once we note that the density of $W$ bosons at criticality is exponentially small due to the Boltzmann factor suppression. Thus, $W$ 's can be treated in the dilute gas approximation in the same way as in the $\mathrm{SU}(2)$ theory. To do this explicitly we first have to understand how to write the partition function in the presence of one $W$ boson of a particular type 53

Let us first consider a $W$ boson corresponding to one of the fundamental roots $\beta_{k}$. Combining Eq. 201) with (214) and (220) we see that this $W$ boson corresponds to unit vorticity of the field $V_{k}$ and zero vorticity of all other fields 
$V_{j}, j \neq k$. To create such a vortex in the path integral we must introduce an external "current" which forces the discontinuity of the field $\chi_{k}$

$$
\chi_{k}=\chi_{k}+2 \pi .
$$

The partition function in the presence of one $W$ boson is thus

$$
Z=\int D[\chi(x)] \exp \left\{-\int d^{2} y\left(S[A]+\sum_{\alpha} \zeta_{\alpha} \cos \left(2 \sum_{i} \vec{\alpha} \cdot \vec{\beta}_{i} \chi_{i}\right)\right)\right\}
$$

where

$$
S[A]=\frac{g^{2}}{16 \pi^{2} T} \sum A_{i j}\left(\partial_{\mu} \chi_{i}-J_{\mu}^{i}(y, x)\right)\left(\partial_{\mu} \chi_{j}-J_{\mu}^{j}(y, x)\right),
$$

where

$$
J_{\mu}^{i}(y, x)=2 \pi \delta_{i k} n_{\mu}(y) \delta\left(y \in C_{x}\right)
$$

with $C_{x}$ a curve that starts at the location of the vortex (the point $x$ ), and goes to infinity, and $n_{\mu}$ is the unit normal to this curve. The insertion of this current forces the normal derivative of $\chi_{k}$ to diverge on curve $C$, so that $\chi_{k}$ jumps by $2 \pi$ across $C$. Since in the rest of the space $\chi_{k}$ is smooth, the path integral is dominated by a configuration with unit vorticity of $\chi_{k}$.

The path integral Eq. (224) differs from the partition function in the vacuum sector by the linear term in the Lagrangian

$$
-\frac{g^{2}}{4 \pi^{2} T} \sum_{i, j} \int d^{2} y \vec{\beta}_{i} \cdot \vec{\beta}_{j} \partial_{\mu} \chi_{i} J_{\mu}^{j}=-\frac{g^{2}}{4 \pi T} \int_{C_{x}} d x_{\mu} \epsilon_{\mu \nu} \partial_{\nu} \overrightarrow{\beta_{k}} \cdot \vec{\eta} .
$$

Defining in the standard way the dual field $\tilde{\eta}$,

$$
i \partial_{\mu} \overrightarrow{\tilde{\eta}}=\epsilon_{\mu \nu} \partial_{\nu} \vec{\eta}
$$

we can recast the contribution of this particular $W$ boson in the form of the following extra term in the Lagrangian

$$
-i \frac{g^{2}}{4 \pi T} \overrightarrow{\beta_{k}} \cdot \overrightarrow{\tilde{\eta}}
$$

This procedure can be repeated for a $W$ boson corresponding to an arbitrary root $\alpha$ with the only difference that in Eq. (228), the root $\beta_{k}$ is replaced by the root $\alpha$. To create several $W$ bosons one just inserts the external current which is the sum of the currents creating individual $W$ 's. 
A dilute ensemble of such objects with small fugacities $\mu_{\alpha}$ then has

$$
Z=\prod_{\alpha} \sum_{n, m} \frac{1}{n !} \frac{1}{m !} \mu_{\alpha}^{n+m} \int \prod_{i} d x_{i} \prod_{j} d y_{j} Z\left(x_{i}, y_{j}\right) .
$$

The result of the summation over the number of $W$ 's is the partition function with the Lagrangian

$$
\mathcal{L}_{\text {eff }}=\frac{g^{2}}{32 \pi^{2} T}\left(\partial_{\mu} \vec{\eta}\right)^{2}+\sum_{\alpha} \zeta_{\alpha} \exp (i \vec{\alpha} \cdot \vec{\eta})+\sum_{\alpha} \mu_{\alpha} \exp \left(i \frac{g^{2}}{4 \pi T} \vec{\alpha} \cdot \overrightarrow{\tilde{\eta}}\right)
$$

with summation in both terms going over all non-vanishing roots of $\mathrm{SU}(N)$. The coefficients $\mu_{\alpha}$ are proportional to the fugacities of the corresponding $W$ bosons

$$
\mu_{\alpha} \propto \exp \left\{-M_{W_{\alpha}} / T\right\} .
$$

Equation (230) is the dimensionally reduced theory which we will now use to study the phase transition.

Let us first disregard the $W$ boson induced term in the effective Lagrangian. If we do that, we are back to the theory of Eq. (221). An interesting property of this theory is that since the group is simply laced (all the roots are of unit length) the anomalous dimensions of all the interaction terms are equal. The scaling dimension of all the monopole induced terms is

$$
\Delta_{M}=\frac{4 \pi T}{g^{2}} .
$$

This immediately tells us that at the temperature

$$
T_{\mathrm{BKT}}=\frac{g^{2}}{2 \pi},
$$

all these interactions become irrelevant. Thus, at $T_{\mathrm{BKT}}$ one expects the BerezinskyKosterlitz-Thouless transition to take place. Above this temperature the infrared behavior of the theory is that of $N-1$ free massless particles. Note that $T_{\mathrm{BKT}}$ does not depend on the number of colors $N$. If the picture just described were true, the universality class of the phase transition would be that of $\mathrm{U}^{N-1}(1)$.

Of course, this is exactly the same situation as encountered in Ref. 25 in the $\mathrm{SU}(2)$ case. Again just like in $\mathrm{SU}(2)$ case this conclusion is incorrect due to the contribution of the $W$ bosons. To see this, it is simplest to ask what would happen at high temperature if there were no monopole contributions at all. This amounts to studying Eq. (230) with $\xi_{\alpha}=0$. This theory describes 
non-compact electrodynamics with $N-1$ photons and the spectrum of charged particles given by Eq. (201). This limit is again simple to understand, since the theory is exactly dual to the theory with monopoles and without charges. The scaling dimensions of all the $W$ induced perturbations are equal and are given by

$$
\Delta_{W}=\frac{g^{2}}{4 \pi T} .
$$

Thus, the perturbations are irrelevant at low temperature, but become relevant at

$$
T_{N C}=\frac{g^{2}}{8 \pi} .
$$

Since $T_{N C}<T_{\mathrm{BKT}}$ this tells us that we can not neglect the effects of charges at criticality. The story of $\mathrm{SU}(2)$ exactly repeats itself. Even the value of the temperature at which the scaling dimensions of the charge - and monopole induced perturbations are equal does not depend on $N$.

We expect therefore that the actual transition temperature is

$$
T_{C}=\frac{g^{2}}{4 \pi}
$$

at which point all perturbations have the same scaling dimension. This expectation is supported by the renormalization group analysis.

The renormalization group equations for the theory Eq. (230) were studied in Ref. 54. In general the equations are quite complicated due to the cross correlations between different operators. For this reason the space of parameters of the theory has to be enlarged if one wants to study the flow whose ultraviolet initial condition is provided by Eq. (230) with arbitrary values of fugacities. However, there is one simple case, that is when the initial condition is such that all the monopole fugacities are equal $\xi_{\alpha_{i}}=\xi_{\alpha_{j}}=\xi$, and all the charge fugacities are equal $\mu_{\alpha_{i}}=\mu_{\alpha_{j}}=\mu$. This initial condition is stable under the renormalization group flow. In this subspace the renormalization group equations, written in terms of the scaled temperature $t=\frac{4 \pi T}{g^{2}}$ and dimensionless fugacities, read

$$
\begin{aligned}
& \frac{\partial t}{\partial \lambda}=2 \pi^{2} N t\left(\mu^{2}-\zeta^{2}\right), \\
& \frac{\partial \mu}{\partial \lambda}=\left(2-\frac{1}{t}\right) \mu-2 \pi(N-2) \mu^{2}, \\
& \frac{\partial \zeta}{\partial \lambda}=(2-t) \zeta-2 \pi(N-2) \zeta^{2} .
\end{aligned}
$$


These equations have exactly the property reflecting our previous discussion. That is the points $t=2, \quad \mu=0$ and $t=1 / 2, \quad \xi=0$ are both unstable. The stable infrared fixed point is

$$
t_{0}=1 \quad \mu_{0}=\zeta_{0}=\frac{1}{2 \pi(N-2)} .
$$

One can in fact easily check that, in the three-dimensional space of couplings $t, \xi$ and $\mu$, this point has two attractive and one repulsive direction. This is precisely what one expects from the infrared fixed point located on the critical surface, the two attractive directions being the tangential directions to the surface.

The renormalization group equations have an obvious duality symmetry, $\mu \rightarrow \xi, \quad t \rightarrow 1 / t$. This is the reflection of the transformation $\eta \rightarrow \tilde{\eta}$ on the level of the Lagrangian 230. The points $t=1, \mu=\xi$ are symmetric under duality, and this ensures the existence of a self dual fixed point. This is important, since the exact position of the fixed point is scheme dependent. Its existence however is ensured by the duality symmetry.

What is the nature of this fixed point? For $N=2$ we were able in Ref. 26 to fermionize the fixed point theory and show explicitly that it is equivalent to one massless Majorana fermion. We are not able to perform a similar analysis for arbitrary $N$. There are however several comments that we would like to make. Phase transitions in $Z_{N}$ invariant spin models have been studied quite extensively. A recent discussion of the situation is given in Ref. 55. One considers a spin model of one phase field $\theta$ with a symmetry breaking term of the type $h \cos \{N \theta\}$ which breaks the $\mathrm{U}(1)$ symmetry down to $Z_{N}$. When the coefficient $h$ of this symmetry breaking term is large, the model resembles the Potts model and thus (for $N>4$ ) has a first order phase transition. When the breaking is small on the other hand, the behavior is similar to the Villain model: the system undergoes two BKT type transitions with a massless U(1) symmetric phase at intermediate temperatures. At some particular "tricritical" value of $h$ the massless phase shrinks to a point and it comes together with the first order transition line. This tricritical point is self-dual and is described by a conformal $Z_{N}$ invariant parafermionic theory with the central charge $c=2(N-1) /(N+2)$ introduced in Ref. 56. In this type of model therefore one generically expects either a first order transition or a pair of BKT transitions with a massless phase in between. The tricritical behavior is special and requires fine tuning of the parameters. This is indeed also the prevailing general expectation for the order of the transition in $2+1$ dimensional gauge theories at large $N$ : either first order or Villain type $\mathrm{U}(1)$ invariant behavior.

In fact, we find in our model a completely different situation. The tran- 
sition is not first order, and there is no $\mathrm{U}(1)$ invariant massless phase. We stress that within the renormalization group flow Eq. (239) the infrared fixed point Eq. (240) has two attractive directions. This means that it governs the infrared behavior of the points which lie on a two-dimensional critical surface in the three-dimensional parameter space, and is therefore generic. This by itself does not preclude the possibility that this fixed point is the same as in the parafermionic $Z_{N}$ theory of Ref. 56. If this is the case, it is quite interesting, since the point which appeared as "tricritical" from the point of view of usual spin models is in fact generic from the point of view of the 3D gauge theories. Although we can not prove that our critical point is described by the parafermionic theory, we will present some arguments supporting this conjecture. The point is that, as opposed to models considered in Ref. 55 our Lagrangian (230) describes a theory of $N-1$ light fields. The theory of $N-1$ free massless fields has the ultraviolet central charge $c_{\mathrm{UV}}=N-1$. However, this conformal field theory is deformed by the monopole and $W$ induced perturbations and flows to a different infrared fixed point. However, let us note that the central charge $c=N-1$ is precisely the central charge of the $\mathrm{SU}(N)_{1}$ Wess-Zumino-Novikov-Witten (WZNW) model. The Ising (i.e. $c=1 / 2$ ) model is the lowest among the minimal models with Virasoro (i.e. $W_{2}$ ) symmetry. The highest model of this class is the $c=1$ model (one free field ) which is precisely the $\mathrm{SU}(2)_{1}$ WZNW model. When the $c=1$ model was deformed by the monopole and $W$-boson operators the central charge was reduced - and the resulting infrared theory was Ising-like.

Now, $Z_{N}$ parafermions with $c=2(N-1) / N+2$ are the lowest minimal models with $W_{N}$ symmetry, and the highest is $\mathrm{SU}(N)_{1}$ (for more information about parafermions see, for example, Ref. 57 and references therein), which can be described in terms of $N-1$ massless fields. Thus, if the theory in the ultraviolet describes $N-1$ massless fields and has $W_{N}$ symmetry, it is quite possible that result of the relevant (monopole $+W$ ) deformation is a selfdual critical point. It is indeed known that the $Z_{N}$ parafermion theory is the self-dual model with $W_{N}$ symmetry. The fact that the central charge (and thus the effective number of degrees of freedom) is reduced in the process of the flow towards the infrared is of course in complete accord with Zamolodchikov's $C$-theorem. It is therefore possible that the infrared fixed point that describes the universality class of the Georgi-Glashow model is the conformal $Z_{N}$ parafermion theory.

Analysis of Ref. 54, although admittedly incomplete also supports the expectation that we do not have the Villain picture. As discussed in detail in Ref. 53, it is the presence of the large number of fields that drives our theory away from the Villain behavior. 
An interesting feature of this result is that the critical temperature in the $\mathrm{SU}(N)$ theory at large $N$ is proportional to the coupling $g^{2}$ and not to the 't Hooft coupling $\lambda=g^{2} N$. Thus, at large $N$ the critical temperature approaches zero. The physical reason for this is easy to understand. At large $N$ and fixed $\lambda$ the Higgs VEV should also scale with $N$ in such a way that the mass of $W$ bosons remains fixed. The monopole action then grows as $N$ and the photons get progressively lighter (exponentially with $N$ ) . Thus, the thickness of the confining string grows and the density of $W$ bosons needed to restore the symmetry becomes smaller and smaller.

More importantly, our main conclusion is that the deconfining transition in the $\mathrm{SU}(N)$ Georgi-Glashow model is second order and the universality class is determined by the infrared fixed point Eq. (240). This point is $Z_{N}$ symmetric and self dual. We have given some arguments supporting the possibility that the fixed point theory is the $Z_{N}$ parafermionic model56 although we are not able to prove this explicitly. We can however definitely exclude the Potts and Villain universality classes.

\section{Concluding remarks}

We have discussed at length various aspects of the confining physics and the deconfining phase transition in weakly interacting gauge theories in $2+1$ dimensions. Naturally one should ask: where do we go from here? The ultimate goal we have in mind is of course strongly interacting $3+1$ dimensional gauge theories. But this goal is still very far away. Many aspects of our discussion would change with dimensionality. In $3+1$ dimensions monopoles are not instantons any longer, but particles, while the magnetic vortices are not particles but strings. The analog of the effective low-energy theories we have discussed, which incorporate the effects of these objects is not known. Moreover, weakly coupled phases of gauge theories are generically not confining and separated from the confining ones by phase transition. Thus, even if such effective theories were known it is not clear to what extent they can be used as a guide for the physics of the confining phase. The situation is not hopeless though, and a lot of work is being done on $3+1$ dimensional theories. In particular, the dual superconductivity picture within the Abelian dominance hypothesis has been studied quita extensively 58 More recently the vortex-based ideas have also gained support 59

\footnotetext{
${ }^{i}$ This is analogous to the situation in QCD where the instantons become less relevant at large $N$ and the $\eta^{\prime}$ meson becomes massless. The major difference is of course that while the $\eta^{\prime}$ mass in QCD decreases as $1 / N$, the photon masses in Georgi-Glashow model decrease exponentially. This difference is due to the non diluteness of the instanton gas in QCD as opposed to diluteness of the monopole gas in the Georgi-Glashow model.
} 
Perhaps a simpler question is what can one say about the strongly interacting regime in $2+1$ dimensions. Here some progress has been made. Both monopole based 60 and vortex based approaches 12 have been advanced. The basic encouraging feature is that the lightest degrees of freedom seem to be the same in the strongly and weakly coupled regimes, This also seems to be the case near the deconfining transition temperature 53 Thus, a certain continuity is there and one hopes eventually to learn how to utilize it to the full extent.

In short, the problems are hard — but don't despair ...

\section{Acknowledgments}

We thank our collaborators Cesar Fosco, Chris Korthals Altes, Baruch Rosenstein, Martin Schvellinger, Ben Svetitsky and Bayram Tekin for collaboration and discussions on subjects covered in this review. We thank Ben Gripaios for reading the manuscript. This work has been supported partly by PPARC.

0.

1. A.M. Polyakov, Phys. Lett. B 59, 82 (1975); Nucl. Phys. B 120, 429 (1977).

2. G. Bhanot and B.A. Freedman, Nucl. Phys. B 190, 357 (1981); Y. Munehisa, Phys. Lett. B 155, 159 (1985); G.N. Obodi, Phys. Lett. B 174, 208 (1986); M.G. Amaral and M.E. Pol, J. Phys. G: Nucl. Part. Phys. 16, 1 (1990).

3. A. Kovner, in M. Shifman, (Ed.) At the Frontier of Particle Physics, Handbook of QCD/Ioffefestschrift, (World Scientific, Singapore, 2001) Vol. 3, pp. 1777 - 1825 hep-ph/0009138.

4. E. Fradkin and S.H. Shenker, Phys. Rev. D 19, 3682 (1979).

5. M. K. Prasad and C. M. Sommerfield,Phys. Rev. Lett. 35, 760 (1975); E. B. Bogomolny, Sov. J. Nucl. Phys. 24, 861 (1976); T. W. Kirkman and C. K. Zachos, Phys. Rev. D 24, 999 (1981).

6. S. Coleman, Phys. Rev. D 11, 2088 (1975); S. Samuel, Phys. Rev. D 18, 1916 (1978); D. J. Amit, Y. Y. Goldschmidt and G. Grinstein, J. Phys. A 15585 (1980); D. Boyanovsky, J. Phys. A 22 2601 (1989).

7. A.M. Polyakov, Gauge Fields and Strings, (Harwood Academic Publishers, Chur, Switzerland, 1987).

8. G. 't Hooft, Nucl. Phys. B 138, 1 (1978), Acta Phys. Austr. Suppl. XXII (1980) 531.

9. S. Samuel, Nucl. Phys. B 154, 62 (1979) 
10. A. Kovner and B. Rosenstein, Int. J. Mod. Phys. A 7, 7419 (1992).

11. C.P. Korthals-Altes and A. Kovner, Phys. Rev. D 62, 096008 (2000)

12. A. Kovner and B. Rosenstein, J. High Energy Phys. 003, 9809 (1998).

13. J. Ambjorn and J. Greensite, J. High Energy Phys. 004, 9805 (1998).

14. A.M. Polyakov, Nucl. Phys. B 486, 23 (1997), hep- th/9607049.

15. M.C. Diamantini, F.Quevedo and C.A. Trugenberger, Phys. Lett. B 396, 115 (1997) hep-th/9612103]; D. Antonov, Phys. Lett. B 427, 274 (1998) hep-th/9804016]; Phys. Lett. B 428, 346 (1998) hep-th/9802056.

16. A.M. Polyakov, Nucl. Phys. B 268, 406 (1986); H. Kleinert, Phys. Lett. B 174, 335 (1986).

17. M.C. Diamantini, H.Kleinert, C.A. Trugenberger, Phys. Lett. B 457, 87 (1999), hep-th/9903208

18. V.I. Ogievetsky, I.V. Polubarinov, Sov. J. Nucl. Phys. 4156 (1967), Yad. Fiz. 4216 (1966); M. Kalb, P. Ramond, Phys. Rev. D 9, 2273 (1974); D.Z. Freedman, P.K. Townsend, Nucl. Phys. B 177, 282 (1981).

19. I.I. Kogan, A. Kovner and M. Schvellinger J. High Energy Phys. 0107, 019 (2001) hep-th/0103235.

20. C. Fosco and A. Kovner, Phys. Rev. D 63, 045009 (2001) hepth/0010064.

21. I. Kogan and A. Kovner Phys. Rev. D 51, 1948 (1995) hep-th/9408081].

22. A. Kovner and B. Svetitsky, Phys. Rev. D 60, 105032 (1999) heplat/9811015.

23. E.C. Marino, Phys. Rev. D 38, 3194 (1998); A. Kovner, B. Rosenstein and D. Eliezer, Nucl. Phys. B 350, 325 (1991).

24. S. D. Drell, H. R. Quinn, B. Svetitsky, and M. Weinstein, Phys. Rev. D 19, 619 (1979).

25. N. O. Agasian and K. Zarembo, Phys. Rev. D 57, 2475 (1998) hepth/9708030. For the $\mathrm{SU}(N)$ theory similar analysis omitting the effects of $W$ bosons was performed recently in N.O. Agasyan and D. Antonov, hep-th/0104029.

26. G. Dunne, I. I. Kogan, A. Kovner and B. Tekin, J. High Energy Phys. 0101, 032 (2001) hep-th/0010201.

27. M.N. Chernodub, E.-M. Ilgenfritz, A. Schiller Phys. Rev. D 64, 054507 (2001); Phys. Rev. D 64, 114502 (2001) hep-lat/0106021.

28. V.L. Berezinskii, Sov. Phys. JETP 32493 (1971); J.M. Kosterlitz and D.J. Thouless, J. Phys. C7 1181 (1973).

29. B. Svetitsky and L.G. Yaffe, Nucl. Phys. B 210, 423 (1982).

30. J.V. José, L.P. Kadanoff, S. Kirkpatrick and D.R. Nelson, Phys. Rev. D 16, 1217 (1977);

A. P. Young, J. Phys. C11 L4553 (1978); 
K. Huang and J. Polonyi, Int. J. Mod. Phys. A 6, 409 (1991).

31. D.G. Shelton, A.A. Nersesyan and A.M. Tsvelik, Phys. Rev. B 64, 8521 (1996).

32. A.O.Gogolin, A.A. Nersesyan and A.M. Tsvelik, Bosonization and Strongly Correlated Systems, (Cambridge University Press, 1998).

33. C.P. Korthals-Altes, A. Kovner and M. Stephanov, Phys. Lett. B 469, 205 (1999).

34. I. I. Kogan, A. Kovner and B. Tekin, J. High Energy Phys. 0103, 021 (2001) [hep-th/0101171].

35. R. Hagedorn, Nuovo Cimento 3, 147 (1965).

36. S. Fubini and G. Veneziano, Nuovo Cimento 64A, 1640 (1969);

K. Huang and S. Weinberg, Phys. Rev. Lett. 25, 895 (1970).

37. S. Frautschi, Phys. Rev. D 3, 2821 (1971);

R.D. Carlitz, Phys. Rev. D D5, 3231 (1972).

38. N. Cabibbo and G. Parisi, Phys. Lett. B 59, 67 (1975).

39. J. Polchinski, String Theory, Vols. 1, 2, (Cambridge University Press, 1998).

40. S.A. Abel, J.L.F. Barbón, I.I. Kogan and E. Rabinovici, J. High Energy Phys. 9904, 015 (1999) hep-th/9911004.

41. I.I. Kogan, JETP Lett. 45709 (1987) [Pisma Zh. Eksp. Teor. Fiz. 45 556 (1987)].

42. B. Sathiapalan, Phys. Rev. D 35, 3277 (1987).

43. J.J. Atick, E. Witten, Nucl. Phys. B 310, 291 (1988).

44. A.A. Abrikosov, Jr., I.I. Kogan, Sov.Phys.JETP 69235 (1989); Int. J. Mod. Phys. A 1501, 1991 (.)

45. B. Sathiapalan, Mod. Phys. Lett. A 13, 2085 (1998) hep-th/9805126.

46. D.A. Lowe, L. Thorlacius, Phys. Rev. D 51, 665 (1995) [hepth/9408134; S. Abel, K. Freese, I. I.Kogan, J. High Energy Phys. 0101, 039 (2001) hep-th/0005028].

47. I.I. Kogan, J.F Wheater, Phys. Lett. B 403, 31 (1997) hep-th/9703141.

48. F. Englert and P. Windey, Phys. Rev. D 14, 2728 (1976).

49. P. Goddard, J. Nuyts and D. Olive, Nucl. Phys. B 125, 1 (1977).

50. E. J. Weinberg, Nucl. Phys. B 167, 500 (1980).

51. S. R. Wadia and S. R. Das, Phys. Lett. B 106, 386 (1981).

52. N. J. Snyderman, Nucl. Phys. B 218, 381 (1983).

53. I.I. Kogan, A. Kovner and B. Tekin, J. High Energy Phys. 0105, 062 (2001) hep-th/0104047.

54. D. Boyanovsky and R. Holman, Nucl. Phys. B 358, 619 (1991).

55. P. Dorey, R. Tateo and K.E. Thompson, Nucl. Phys. B 470, 317 (1996) hep-th/9501098. 
56. A.B. Zamolodchikov and V.A. Fateev, Sov.Phys. JETP 62215 (1985); V.A. Fateev and A.B. Zamolodchikov, Nucl. Phys. B 280, 644 (1987); D.Gepner and Z. Qiu, Nucl. Phys. B 285, 423 (1987).

57. A. Cappelli, L.S. Georgiev and I.T.Todorov, Nucl. Phys. B 559, 499 (2001) hep-th/0009229.

58. For a recent review see A. Di Giacomo, hep-lat/0112002.

59. M. Faber, J. Greensite and S. Olejnik J. High Energy Phys. 0006, 041 (2000) [hep-lat/0005017].

60. S. R. Das and S. R. Wadia, Phys. Rev. D 53, 5856 (1996) hepth/9503184. 